ARTICLE

\title{
Targeting the differential addiction to anti-apoptotic BCL-2 family for cancer therapy
}

Akane Inoue-Yamauchi ${ }^{1} \uparrow$, Paul S. Jeng ${ }^{1}$, Kwanghee Kim², Hui-Chen Chen ${ }^{1}$, Song Han¹, Yogesh Tengarai Ganesan', Kota Ishizawa1, Sylvia Jebiwott², Yiyu Dong1, Maria C. Pietanza3,4, Matthew D. Hellmann³, Mark G. Kris ${ }^{3,4}$, James J. Hsieh ${ }^{5}$ \& Emily H. Cheng $1,6,7$

BCL-2 family proteins are central regulators of mitochondrial apoptosis and validated anticancer targets. Using small cell lung cancer (SCLC) as a model, we demonstrated the presence of differential addiction of cancer cells to anti-apoptotic BCL-2, BCL- $X_{L}$ or $M C L-1$, which correlated with the respective protein expression ratio. $A B T-263$ (navitoclax), a BCL-2/BCL-X inhibitor, prevented $B C L-X_{L}$ from sequestering activator $B H 3$-only molecules (BH3s) and $B A X$ but not BAK. Consequently, ABT-263 failed to kill BCL- $\mathrm{X}_{\mathrm{L}}$-addicted cells with low activator $\mathrm{BH} 3 \mathrm{~s}$ and $B C L-X_{L}$ overabundance conferred resistance to $A B T-263$. High-throughput screening identified anthracyclines including doxorubicin and CDK9 inhibitors including dinaciclib that synergized with ABT-263 through downregulation of MCL-1. As doxorubicin and dinaciclib also reduced $B C L-X_{L}$, the combinations of $B C L-2$ inhibitor ABT-199 (venetoclax) with doxorubicin or dinaciclib provided effective therapeutic strategies for SCLC. Altogether, our study highlights the need for mechanism-guided targeting of anti-apoptotic BCL-2 proteins to effectively activate the mitochondrial cell death programme to kill cancer cells.

\footnotetext{
${ }^{1}$ Human Oncology and Pathogenesis Program, Memorial Sloan Kettering Cancer Center, 1275 York Avenue, New York, New York 10065, USA. ${ }^{2}$ Department of Surgery, Memorial Sloan Kettering Cancer Center, New York, New York 10065, USA. ${ }^{3}$ Thoracic Oncology Service, Department of Medicine, Memorial Sloan Kettering Cancer Center, New York, New York 10065, USA. ${ }^{4}$ Department of Medicine, Weill Cornell Medical College, New York, New York 10065 , USA. ${ }^{5}$ Molecular Oncology, Department of Medicine, Siteman Cancer Center, Washington University, St Louis, Missouri 63110, USA. ${ }^{6}$ Department of Pathology, Memorial Sloan Kettering Cancer Center, New York, New York 10065, USA. 7 Department of Pathology and Laboratory Medicine, Weill Cornell Medical College, Cornell University, New York, New York 10065, USA. † Present address: Department of Pathology, Tokyo Women's Medical University, Shinjuku-ku, Tokyo 162-8666, Japan. Correspondence and requests for materials should be addressed to E.H.C. (email: chenge1@mskcc.org).
} 
T he BCL-2 family proteins dictate cellular survival or death decisions by regulating the integrity of the mitochondrial outer membrane $(\mathrm{MOM})^{1}$. Apoptotic signals culminate in MOM permeabilization (MOMP), prompting the release of cytochrome $c$ and the activation of caspases. Initiation of the BCL-2 regulated cell death cascade occurs through the transcriptional and posttranslational activation of proapoptotic BH3-only molecules (BH3s), which serve as death sentinels that either directly activate multidomain proapoptotic $\mathrm{BAX}$ and $\mathrm{BAK}$ ('activator' BH3s) or inactivate multidomain anti-apoptotic BCL$2, \mathrm{BCL}-\mathrm{X}_{\mathrm{L}}$ and MCL-1 ('inactivator' BH3s) ${ }^{2-6}$. BAX and BAK, the essential effectors of MOMP, undergo stepwise, bimodal conformational changes upon activation by the activator $\mathrm{BH} 3 \mathrm{~s}$ to form homo-oligomers that mediate cytochrome $c$ efflux ${ }^{4,6,7}$. Conversely, anti-apoptotic BCL-2, BCL- $\mathrm{X}_{\mathrm{L}}$ and MCL-1 preserve mitochondrial integrity through sequestration of activator $\mathrm{BH} 3 \mathrm{~s}$ or partially activated, $\mathrm{BH} 3$-exposed, $\mathrm{BAX} / \mathrm{BAK}$ monomers to prevent the homo-oligomerization of $\mathrm{BAX} / \mathrm{BAK}^{1-4,6,8}$. To evade apoptotic checkpoints, cancer cells often overexpress antiapoptotic BCL-2 proteins ${ }^{9}$. Consequently, along with the fact that activation of the BCL-2-controlled apoptotic pathway seems critical for the efficacy of most chemotherapeutics, BCL-2 family members have emerged as attractive targets for therapeutic development.

Structure-based efforts led to the development of the first specific small molecule inhibitor of the BCL-2 family, ABT-737 and its orally bioavailable analog ABT-263 (navitoclax) that bind and inhibit BCL-2, BCL- $\mathrm{X}_{\mathrm{L}}$ and BCL-W, but not MCL-1 or BCL2A1 (refs 10-14). Although navitoclax showed promising clinical activity, it induced a dose-dependent thrombocytopenia as an on-target result of BCL- $\mathrm{X}_{\mathrm{L}}$ inhibition ${ }^{15,16}$. This spurred the development of ABT-199 (venetoclax or GDC-0199), a plateletsparing, selective BCL-2 inhibitor ${ }^{17}$. Venetoclax has exhibited remarkable therapeutic efficacy for relapsed/refractory chronic lymphocytic leukaemia with an overall response rate of $79 \%$ (ref. 18), resulting in its approval by the Food and Drug Administration (FDA) for the treatment of chronic lymphocytic leukaemia patients with $17 \mathrm{p}$ deletion. Selective inhibitors for BCL- $\mathrm{X}_{\mathrm{L}}$ with robust preclinical activity have also been generated $^{19}$, but similar efforts to target MCL-1 have been less successful. The lack of effective MCL-1 inhibitors positions MCL1 as a key primary as well as secondary resistance factor to ABT263 and ABT-199.

Small cell lung cancer (SCLC) is an aggressive type of neuroendocrine carcinoma that represents $10-15 \%$ of all lung cancer malignancies ${ }^{20}$. Standard first-line treatment consists of a combined regimen of platinum-based chemotherapy with etoposide and typically elicits high initial response rates, followed by almost universal disease recurrence and progression $^{21}$. As a result, 5-year survival rate is dismal $(\sim 5 \%)$ with little improvement over the past 30 years $^{20,21}$. Unlike nonSCLC, which is commonly associated with targetable kinase mutations, SCLC biology is less evidently tractable, driven instead by nearly uniform loss of tumour suppressors TP53 and RB1 (refs 22,23). Preclinical studies showed that SCLC cell lines are among the most sensitive tumour types to ABT-737 and ABT-263 (refs 10,11,24,25), suggesting that targeting the BCL-2 family proteins may be a paradigm shifting therapeutic strategy for this cancer. However, not all SCLC cell lines are sensitive to ABT-263 (refs $11,24,25)$ and limited single agent activity of navitoclax was observed in a phase II trial for SCLC ${ }^{16}$. It has become evident that combination therapy with ABT-263 is required to improve the therapeutic outcome of SCLC. However, it remains unclear how ABT-263 can be integrated with existing chemotherapeutics into rational combination treatments for SCLC, or if particular classes of targeted therapeutics will synergize favourably with ABT-263.
Moreover, reliable biomarkers for identifying patient populations who will respond to ABT-263 monotherapy are yet unknown.

Using an unbiased high-throughput screening (HTS) strategy, we identified anthracyclines including doxorubicin and CDK9 inhibitors including dinaciclib that enhanced the proapoptotic effect of ABT-737/263 through downregulation of MCL-1. Both doxorubicin and dinaciclib also cooperated with ABT-199 to induce robust apoptosis, because they also partially reduced BCL- $\mathrm{X}_{\mathrm{L}}$. The in vivo therapeutic efficacy of these combinations was demonstrated in mouse xenograft models, validating new potential therapeutic strategies for SCLC. Interestingly, we found that some SCLC cell lines displayed differential addiction to BCL-2, BCL-X $X_{L}$ or MCL-1 for survival, which could be determined by the respective protein expression ratio. Surprisingly, ABT-263 failed to kill BCL-X $\mathrm{X}_{\mathrm{L}}$-addicted cells with low expression of activator BH3s, as ABT-263 failed to prevent BCL$\mathrm{X}_{\mathrm{L}}$ from sequestering $\mathrm{BAK}$ in these cells. Consequently, overexpression of BCL- $\mathrm{X}_{\mathrm{L}}$ conferred resistance to ABT-263, representing a previously unknown therapeutic limitation. Together, our data establish a predictive paradigm for determining SCLC addiction to anti-apoptotic BCL-2 family members and highlight the need for mechanism-guided targeting of anti-apoptotic BCL-2 proteins for effective apoptosis induction.

\section{Results}

HTS identifies anthracyclines that cooperate with ABT-263. To improve the therapeutic outcome of ABT-263 for SCLC, we sought to identify the best combination strategies that enhance the proapoptotic effect of ABT-737/263. HTS of FDA-approved anti-cancer agents was performed to identify agents that cooperate with ABT-737 to kill ABT-737-resistant SCLC. As reported $^{10}$, DMS53 was sensitive whereas H196 was resistant to ABT737 (Fig. 1a). Interestingly, H196 was also resistant to the chemotherapeutic agents used to treat SCLC (Fig. 1a). To explore the mechanisms underlying the differential responses of H196 versus DMS53, the levels of BCL-2 family proteins were assessed. The high expression of MCL-1 and low expression of BCL-2 in H196 cells were probably accountable for their resistance to ABT737 (Fig. 1b). Supporting this notion, knockdown of $M C L-1$ sensitized H196 cells to ABT-737 (Supplementary Fig. 1). In addition, H196 expressed lower levels of BID, BIM and PUMA than DMS53 (Fig. 1b), which might contribute to their resistance to ABT-737 and chemotherapeutic agents as reported ${ }^{5}$.

We next performed HTS of 76 FDA-approved anti-cancer agents to identify agents that cooperate with ABT-737 to kill H196 cells (Fig. 1c and Supplementary Data 1). Effector concentration for half-maximum responses (EC50) of each compound from the FDA panel with or without ABT-737 was determined in H196 cells. Nine compounds from the FDA panel were synergistic with ABT-737, resulting in a greater than twofold decrease of EC50 (Fig. 1d). Five of the nine hits were anthracyclines including doxorubicin (Fig. 1d). As doxorubicin has been included in chemotherapeutic regimens for $\operatorname{SCLC}^{21}$, we subsequently focused on doxorubicin. The combination of ABT737 and doxorubicin indeed induced robust apoptosis in H196 cells (Fig. 1e). In contrast, other chemotherapeutic agents used in treating SCLC, including etoposide, cisplatin and camptothecin, failed to induce comparable apoptosis as doxorubicin when combined with ABT-737 (Fig. 1e). These data strongly argue that ABT-737 does not simply lower the apoptotic threshold of standard cytotoxic agents. In fact, only doxorubicin reduced MCL-1 expression (Fig. 1f), which would sensitize H196 cells to ABT-737 based on the MCL-1 knockdown experiments (Supplementary Fig. 1). 
a

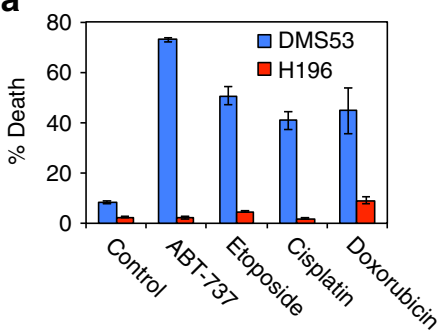

C

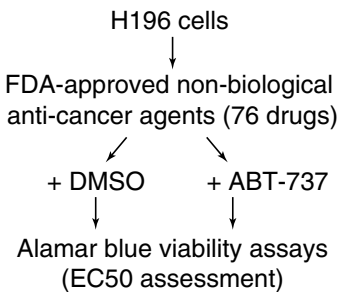

e

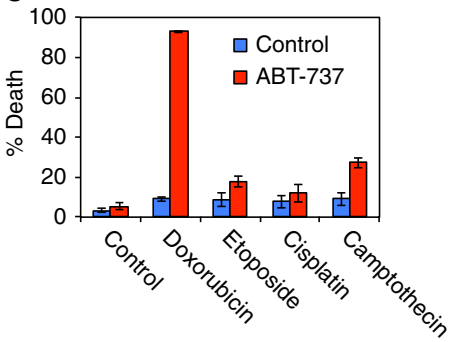

b

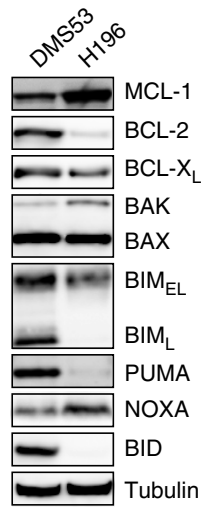

h

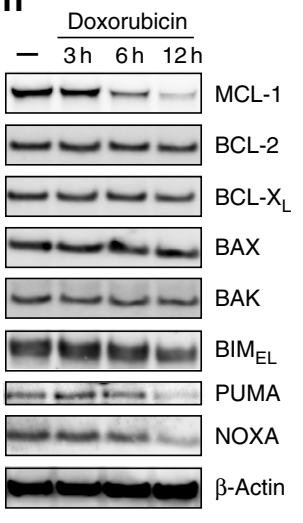

d

\begin{tabular}{l|c|c|l}
\hline Compound & EC50 & $\begin{array}{c}\text { EC50 } \\
\text { with ABT-737 }\end{array}$ & \multicolumn{1}{c}{ Class } \\
\hline Dactinomycin & NA & 0.07 & Antibiotics \\
Vincristine & 0.37 & 0.11 & Mitotic inhibitor \\
Idarubicin & NA & 0.16 & Anthracycline \\
Daunorubicin & NA & 0.45 & Anthracycline \\
Doxorubicin & NA & 0.49 & Anthracycline \\
Mitoxantrone & 1.94 & 0.61 & Anthracycline \\
Epirubicin & NA & 0.63 & Anthracycline \\
Azacitidine & NA & 1.72 & Cytidine analogue \\
Topotecan & NA & 2.99 & Topoisomerase I inhibitor \\
\hline
\end{tabular}

f

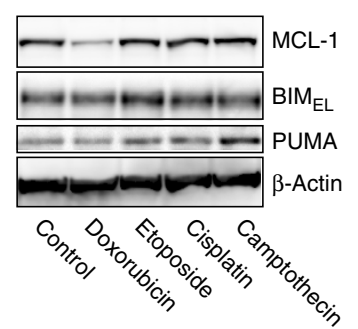

g

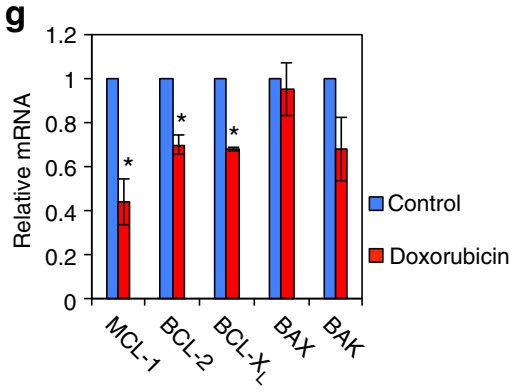

j
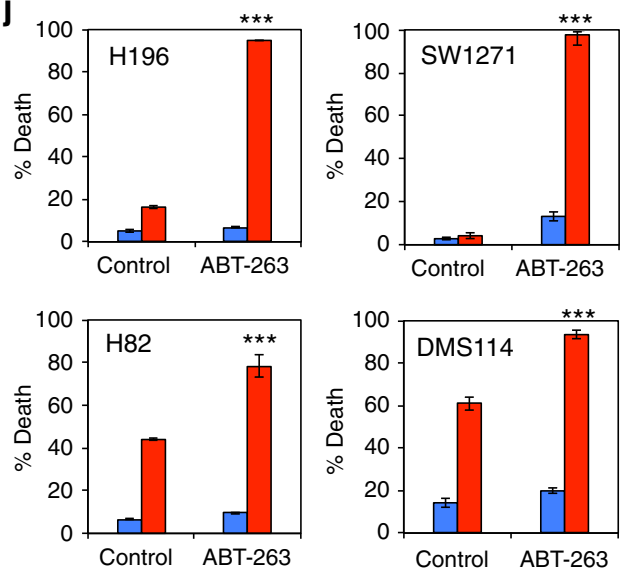
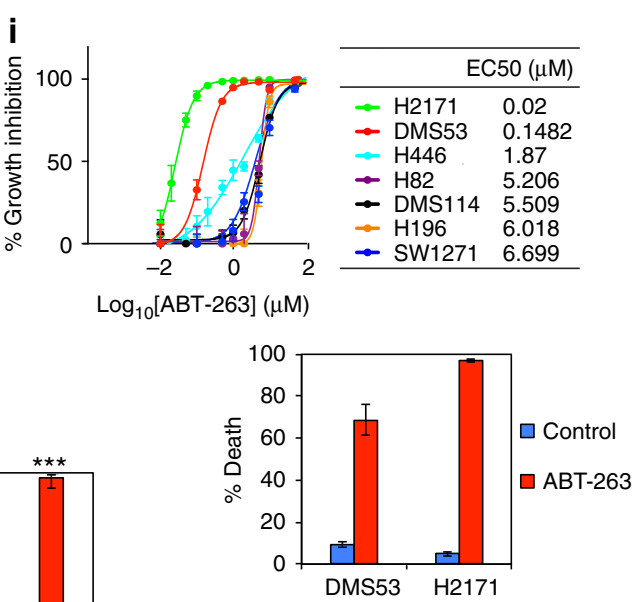

$\square$ Control

$\square$ Doxorubicin

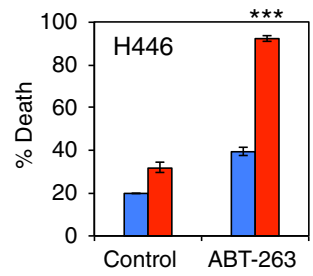

Figure 1 | HTS of FDA-approved anti-cancer agents identifies anthracyclines that enhance the proapoptotic effect of ABT-737. (a) DMS53 and H196 cells were treated with the indicated agents for $48 \mathrm{~h}$. Cell death was quantified by annexin- $V$ staining (mean $\pm s . d$., $n=3$ ). (b) The expression of BCL-2 family proteins in DMS53 and H196 cells was assessed by immunoblot analysis. (c) A schematic of HTS to identify FDA-approved anti-cancer agents that cooperate with ABT-737 to reduce the survival of $\mathrm{H} 196$ cells. $\mathrm{H} 196$ cells were treated with each compound at 12 two-fold serial dilution doses starting from $10 \pm 1 \mu \mathrm{M}$ ABT-737. Cell viability was assessed by Alamar Blue assays at $72 \mathrm{~h}$ and EC50 was calculated. (d) A summary of EC50s of anti-cancer agents \pm ABT-737 in H196 cells. NA denotes 'not applicable', because the agent failed to achieve $100 \%$ growth inhibition at $10 \mu \mathrm{M}$. (e) H196 cells were treated with the indicated agents for $48 \mathrm{~h}$. Cell death was quantified by annexin-V staining (mean \pm s.d., $n=3$ ). (f) H196 treated with the indicated agents for $6 \mathrm{~h}$ were assessed by immunoblot analysis. (g) The mRNA levels of BCL-2 family in H196 cells treated with $2 \mu \mathrm{M}$ doxorubicin for $3 \mathrm{~h}$ were assessed by qRT-PCR. Data were normalized against $\beta$-Actin (mean \pm s.d., $n=2$ independent experiments). (h) H196 cells treated with doxorubicin for the indicated times were assessed by immunoblot analysis. (i) A summary of EC50s of ABT-263 in SCLC cell lines. The indicated SCLC cell lines were treated with increasing concentrations of ABT-263. Cell viability was assessed by CellTiter-Glo assays at $48 \mathrm{~h}$. DMS53 and H2171 cells were treated with $1 \mu \mathrm{M}$ ABT-263 for $24 \mathrm{~h}$ and cell death was quantified by annexin- $V$ staining (mean \pm s.d., $n=3$ ). ( $\mathbf{j}$ ) The indicated SCLC cell lines were treated with $2 \mu \mathrm{M}$ doxorubicin $\pm 1 \mu \mathrm{M}$ ABT-263 for $24 \mathrm{~h}$. Cell death was quantified by annexin-V staining (mean $\pm \mathrm{s} . \mathrm{d} ., n=3$ ). ${ }^{\star} P<0.05$ and ${ }^{\star \star \star} P<0.001$ ( $t$-test). Unprocessed original scans of blots are shown in Supplementary Fig. 11. 
Anthracyclines were reported to be global transcriptional repressors that preferentially affect $M C L-1$ due to its short messenger RNA half-life ${ }^{26}$. We confirmed that doxorubicin rapidly decreased MCL-1 mRNA in H196 cells (Fig. $1 \mathrm{~g}$ and Supplementary Fig. 2). Of note, doxorubicin did not further reduce $M C L-1$ mRNA when combined with actinomycin $\mathrm{D}$, a general transcription inhibitor (Supplementary Fig. 2). Doxorubicin also decreased $B C L-2$ and $B C L-X_{L}$ mRNA but to a lesser extent. Only MCL-1 protein was greatly reduced within $6 \mathrm{~h}$ of doxorubicin treatment, likely to be due to the highly labile nature of MCL-1 protein (Fig. 1h). We subsequently assessed a series of SCLC cell lines for their EC50 to ABT-263 (Fig. 1i). H2171 and DMS53 displayed submicromolar EC50 to ABT-263 and were effectively killed by ABT-263 (Fig. 1i). In contrast, H196, SW1271, H82, DMS114 and H446 were relatively resistant to ABT-263. Importantly, the combination of ABT-263 and doxorubicin induced robust apoptosis in these cell lines (Fig. 1j). In summary, our HTS of the FDA-approved anti-cancer agents identified anthracyclines including doxorubicin as a universal combination strategy with ABT-263 in SCLC.

HTS identifies CDK9 inhibitors that cooperate with ABT-263. Although several targeted therapeutic agents have been demonstrated to enhance the therapeutic effect of ABT-737/263 in preclinical studies, an unbiased approach to identify the best combination strategy for specific cancer types has not been pursued. Accordingly, we extended our HTS to a pathway inhibitor library that is composed of a diverse collection of 993 small molecules against over 200 targets in more than 20 signalling pathways (Supplementary Data 2). H196 cells were treated with each compound from this library with or without ABT-737 (Fig. 2a). Scatterplot analysis of the average percentage growth inhibition identified 11 compounds that conferred more inhibition when combined with ABT-737 (Fig. 2b). Of the 11 hit compounds, 5 were cycline-dependent kinase (CDK) inhibitors that share the common targets of CDK2 and CDK9 (Fig. 2c). PHA-793887 (ref. 27), a CDK inhibitor that mainly targets CDK2, was present in the library but was not identified as a hit. Together, these data suggest that CDK9 is most probably the shared target, which is further supported by the observation that knockdown of CDK9 sensitized H196 cells to ABT-263 (Fig. 2d).

CDK9 is a serine-threonine kinase that forms the catalytic core of $\mathrm{p}$-TEFb complex and, in the presence of cyclin $\mathrm{T}$, phosphorylates Ser2 in the carboxy-terminal domain of RNA polymerase II (Pol II) to stimulate transcription elongation ${ }^{28,29}$. CDK9 inhibitors block transcription elongation and preferentially downregulate transcripts with short half-life such as $M C L-1$ $\mathrm{mRNA}^{30,31}$. Indeed, knockdown of CDK9 reduced MCL-1 in H196 (Fig. 2d). We confirmed that dinaciclib, SNS-032 and AZD5438 reduced the Ser2 phosphorylation of Pol II and synergized with ABT-263 to kill H196 cells (Fig. 2e,f). We subsequently focused on dinaciclib because it is more advanced in clinical development ${ }^{32}$. Dinaciclib rapidly reduced $M C L-1$ mRNA and protein (Fig. 2g,h). The synergistic effect of ABT-263 and dinaciclib in triggering apoptosis was shown in all tested ABT263-resistant SCLC cell lines (Fig. 2i). Of note, PHA-793887 neither suppressed the Ser2 phosphorylation of Pol II nor reduced MCL-1 (Supplementary Fig. 3).

Our HTS identified anthracyclines and CDK9 inhibitors as two combination strategies with ABT-263 through downregulation of MCL-1. Notably, overexpression of MCL-1 protected H196 cells from apoptosis induced by combined ABT-263 and dinaciclib or doxorubicin (Supplementary Fig. 4), supporting that downregulation of MCL-1 is responsible for the observed synergistic proapoptotic effect. Notably, although $>10$ different mammalian target of rapamycin (mTOR) inhibitors were present in our library, none were identified as hits. As it was reported that mTOR inhibitors, including rapamycin and AZD8055, sensitize SCLC cell lines to ABT-737/263 (refs 25,33), we compared the death inducing activity of combined ABT-263 and mTOR inhibitors with that of combined ABT-263 and dinaciclib in four ABT-263-resistant SCLC cell lines (Supplementary Fig. 5). Indeed, combined ABT-263 with dinaciclib was more potent in triggering apoptosis than combined ABT-263 with mTOR inhibitors (Supplementary Fig. 5).

Prediction of cellular addiction to anti-apoptotic BCL-2s. The findings that doxorubicin and dinaciclib downregulated $M C L-1$ and induced apoptosis in H82 and DMS114 as single agents raise the possibility that these cell lines might be dependent on MCL-1 for survival (Figs $1 \mathrm{j}$ and $2 \mathrm{i}$ ). In contrast, DMS53 and $\mathrm{H} 2171$ might be dependent on BCL-2 and/or BCL- $\mathrm{X}_{\mathrm{L}}$ for survival given their sensitivity to ABT-263 (Fig. 1i). DMS114 is unique among the studied SCLC cell lines in that it harbors MCL1 amplification. We hypothesized that a given SCLC cell line might be addicted to a specific anti-apoptotic BCL-2 member for survival if it predominantly expresses that specific anti-apoptotic BCL-2 member. Conversely, a given SCLC cell line that does not predominantly express a specific anti-apoptotic BCL-2 member might not be addicted to any single antiapoptotic BCL-2 member. To test this hypothesis, we first determined whether the seven SCLC cell lines display differential addiction to BCL-2, BCL- $\mathrm{X}_{\mathrm{L}}$ or $\mathrm{MCL}-1$ for survival using RNA interference (RNAi). Knockdown of $M C L-1$ but not $B C L$ 2 or $B C L-X_{L}$ induced significant apoptosis in H82 and DMS114 (Fig. 3a and Supplementary Fig. 6), supporting that both cell lines are addicted to MCL-1 for survival. In contrast, knockdown of $B C L-2$ alone was sufficient to induce significant apoptosis in DMS53 cell line (Fig. 3a and Supplementary Fig. 6), supporting that DMS53 is addicted to BCL-2 for survival. Consistently, ABT-199 effectively killed DMS53 cells (Supplementary Fig. 7). Analogously, H2171 and SW1271 were probably addicted to BCL- $\mathrm{X}_{\mathrm{L}}$ for survival, because knockdown of $B C L-X_{L}$ alone was sufficient to induce significant apoptosis (Fig. 3a and Supplementary Fig. 6). Knockdown of BCL-2, BCL$X_{L}$ or MCL-1 failed to induce significant apoptosis in $\mathrm{H} 196$ and $\mathrm{H} 446$, suggesting that these cell lines are not addicted to any single anti-apoptotic BCL-2 member for survival (Fig. $3 \mathrm{a}$ and Supplementary Fig. 6). Notably, knockdown of both $B C L-X_{L}$ and $M C L-1$ induced robust apoptosis in H196 (Supplementary Fig. 8), which is consistent with its low expression of BCL-2 (Fig. 1b). To this end, we could divide these seven SCLC cell lines into BCL-2-addicted, BCL- $\mathrm{X}_{\mathrm{L}}$-addicted, MCL-1-addicted or non-addicted to any single anti-apoptotic BCL-2 member (Fig. 3a).

We next tested whether selective addiction to BCL-2, BCL- $\mathrm{X}_{\mathrm{L}}$ or MCL-1 for survival is due to the predominant expression of BCL-2, BCL- $\mathrm{X}_{\mathrm{L}}$ or MCL-1 in a given SCLC cell line by assessing the expression of BCL-2 family proteins (Fig. 3b). The expression of BCL-2, BCL- $\mathrm{X}_{\mathrm{L}}$ or MCL- 1 was normalized against $\alpha$-Tubulin and the ratio of an individual anti-apoptotic BCL-2 member to all three members was obtained. We found that the ratio of BCL-2 to combined BCL-2, BCL- $\mathrm{X}_{\mathrm{L}}$ and MCL-1 was highest in BCL-2addicted DMS53, the ratio of $\mathrm{BCL}-\mathrm{X}_{\mathrm{L}}$ was highest in $\mathrm{BCL}-\mathrm{X}_{\mathrm{L}^{-}}$ addicted SW1271 and H2171, and the ratio of MCL-1 was highest in MCL-1-addicted H82 and DMS114 (Fig. 3c). We also compared the mRNA ratios to the protein ratios and found that the mRNA ratios were less predictive than the protein ratios likely due to dysregulated MCL-1 degradation in some cell lines (Supplementary Fig. 9). 
a

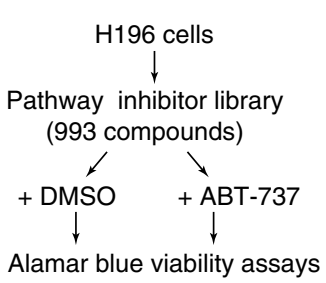

b

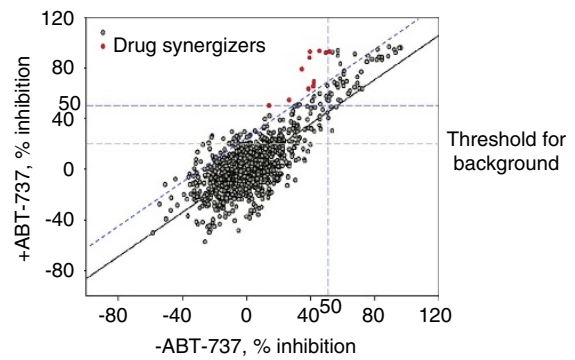

d

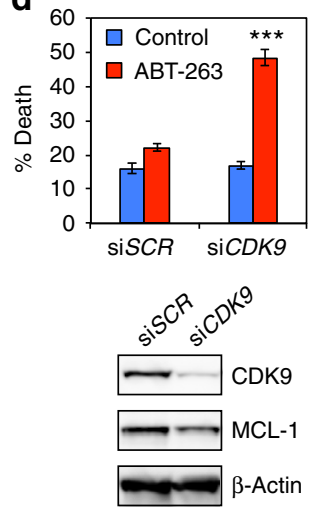

C

\begin{tabular}{|c|c|c|c|}
\hline Compound name & $\begin{array}{c}\text { \% Inhibition } \\
\text { without ABT-737 }\end{array}$ & $\begin{array}{c}\text { \% Inhibition with } \\
\text { ABT-737 }\end{array}$ & Target \\
\hline Depo-(testosterone) & 40 & 93 & Ester of testosterone \\
\hline AT7519 & 52 & 93 & CDK1, 2, 4, 6, 9 \\
\hline Dinaciclib & 45 & 93 & CDK1, 2, 5, 9 \\
\hline Flavopiridol & 50 & 92 & CDK1, 2, 4, 6, 7, 9 \\
\hline SNS-032 & 39 & 88 & CDK2, 7, 9 \\
\hline AZD5438 & 35 & 79 & CDK1, 2, 9 \\
\hline TAK-901 & 42 & 69 & Aurora kinase \\
\hline Epirubicin hydrochloride & 42 & 65 & Topoisomerase \\
\hline KX2-391 & 39 & 63 & Src \\
\hline Tanshinone IIA & 27 & 54 & Diterpene quinone \\
\hline
\end{tabular}

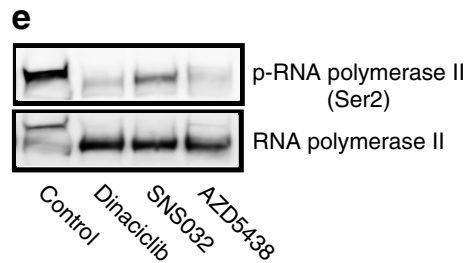

f

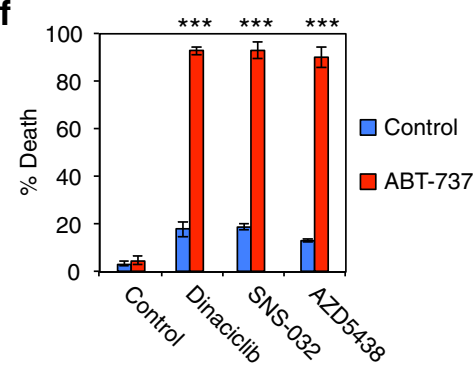

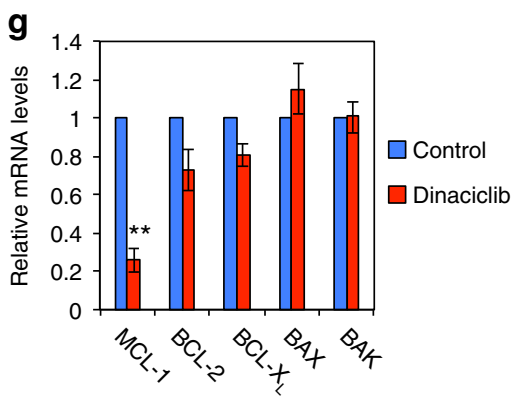
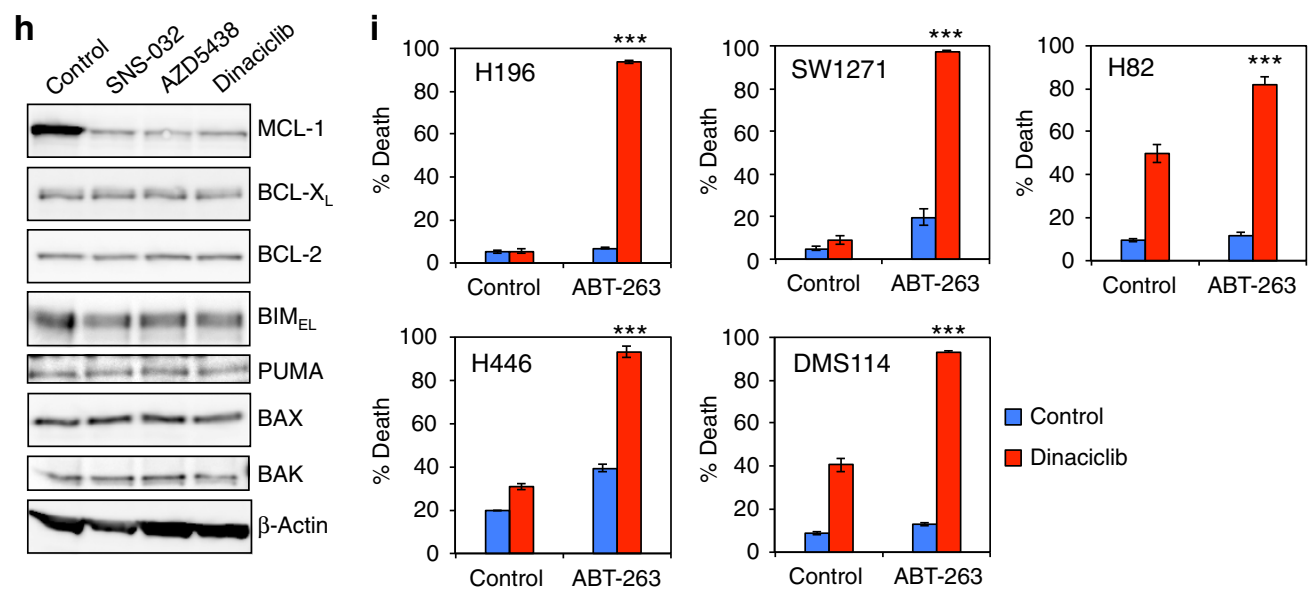

$\square$ Control

$\square$ Dinaciclib

Figure 2 | HTS of a small molecule pathway inhibitor library identifies CDK9 inhibitors that enhance the proapoptotic effect of ABT-737. (a) A schematic of HTS of the pathway inhibitor library to identify agents that cooperate with ABT-737 to kill H196 cells. H196 cells were used to screen the pathway inhibitor library at $2 \mu \mathrm{M} \pm 1 \mu \mathrm{M} \mathrm{ABT-737}$. Compounds were screened in duplicate and cell viability was assessed by Alamar Blue assays at $72 \mathrm{~h}$.

(b) Scatter plot analysis of the average percentage growth inhibition of each compound screened in $\mathrm{H} 196$ cells. The compounds that induced $\leq 50 \%$ growth inhibition in the absence of ABT-737 while $\geq 50 \%$ inhibition in the presence of ABT-737 were identified as positive hits (red dots). (c) A summary of hit compounds identified by the HTS of the pathway inhibitor library. (d) H196 cells, transfected with scrambled siRNA (siSCR) or siRNA against CDK9, were treated with $1 \mu \mathrm{M} \mathrm{ABT}-263$ and subjected to cell death or immunoblot analysis. Cell death was quantified by annexin-V staining (mean $\pm \mathrm{s}$.d., $n=3$ ). (e) H196 cells, treated with the indicated agents for $3 \mathrm{~h}$, were assessed by immunoblot analysis. (f) H196 cells were treated with the indicated agents $\pm 1 \mu \mathrm{M}$ ABT-737 for $24 \mathrm{~h}$. Cell death was quantified by annexin-V staining (mean \pm s.d., $n=3$ ). (g) The mRNA levels of BCL-2 family in H196 cells treated with $10 \mathrm{nM}$ dinaciclib for $3 \mathrm{~h}$ were assessed by qRT-PCR. Data were normalized against $\beta$-Actin (mean \pm s.d., $n=2$ independent experiments). (h) H196 cells, untreated or treated with the indicated CDK9 inhibitors for $6 \mathrm{~h}$, were assessed by immunoblot analysis. (i) The indicated SCLC cell lines were treated with vehicle or dinaciclib (10 nM for $\mathrm{H} 196, \mathrm{H} 82$, and H446, or $20 \mathrm{nM}$ for SW1271 and DMS114) in the absence or presence of $1 \mu \mathrm{M}$ ABT-263 for $24 \mathrm{~h}$. Cell death was quantified by annexin-V staining (mean \pm s.d., $n=3$ ). ${ }^{\star \star} P<0.01$ and ${ }^{\star \star \star} P<0.001$ ( $t$-test). Unprocessed original scans of blots are shown in Supplementary Fig. 11. 
a
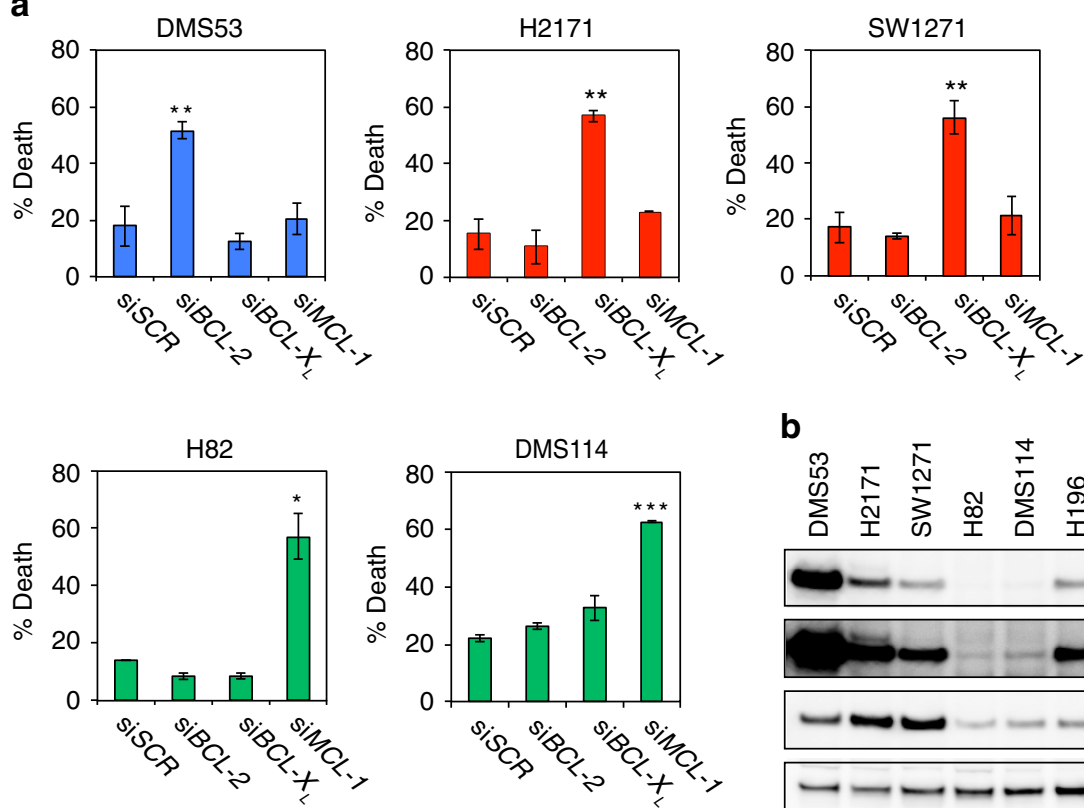

b

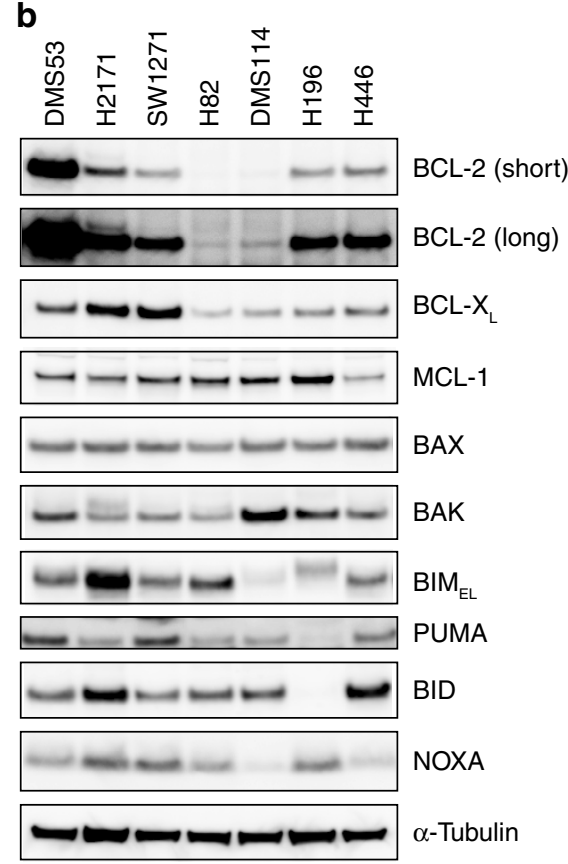

C
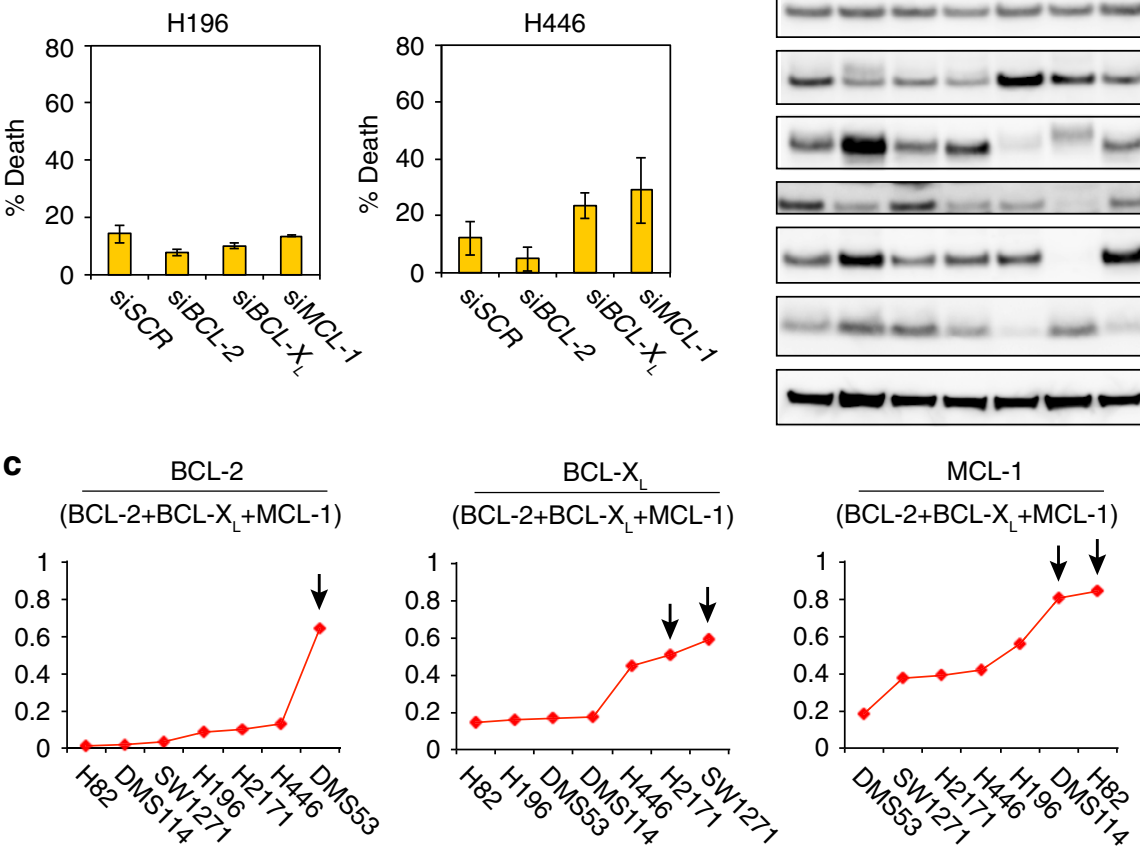

Figure 3 | The SCLC cell lines display differential addiction to anti-apoptotic BCL-2 family proteins for survival. (a) The indicated SCLC cell lines were transfected with scrambled siRNA (siSCR) or siRNA against $B C L-2, B C L-X_{L}$, or $M C L-1$. Cell death was quantified by annexin- $V$ staining (mean $\pm \mathrm{s}$.d., $n=3$ ). (b) The expression of BCL-2 family proteins in the indicated SCLC cell lines was assessed by immunoblot analysis using the indicated antibodies. (c) The protein expression ratios of $B C L-2, B C L-X_{L}$, or $M C L-1$ to combined $B C L-2, B C L-X_{L}$ and $M C L-1$ in the indicated SCLC cell lines. The expression of BCL-2, BCL$X_{L}$, or MCL-1 was normalized against $\alpha$-Tubulin and the ratio of an individual anti-apoptotic $B C L-2$ member to all three members was obtained based on two representative immunoblot analyses. Arrows indicate the cell lines that display addiction to $B C L-2, B C L-X_{L}$ or $M C L-1$ for survival. ${ }^{\star} P<0.05$, ${ }^{\star \star} P<0.01$ and ${ }^{\star \star \star} P<0.001$ (Student's $t$-test). Unprocessed original scans of blots are shown in Supplementary Fig. 11.

ABT-263 fails to kill BCL- $\mathrm{X}_{\mathrm{L}}$-addicted cells with low $\mathrm{BH} 3 \mathrm{~s}$. We have demonstrated that both SW1271 and H2171 were dependent on BCL- $\mathrm{X}_{\mathrm{L}}$ for survival based on the RNAi results (Fig. 3a). However, SW1271 displayed the highest EC50 to ABT-263 whereas H2171 showed submicromolar EC50 to ABT-263 (Fig. 1i). To validate these results, viability was determined by annexin-V staining and ABT-199 was included as a control. ABT-263 but not ABT-199 induced robust apoptosis in H2171 (Fig. 4a). In contrast, ABT-263 only induced mild apoptosis in
SW1271. The dependency of SW1271 on BCL-X $\mathrm{X}_{\mathrm{L}}$ for survival was validated using a second small interfering RNA (siRNA) against $B C L-X_{L}$ (Supplementary Fig. 10). Notably, lower expression of BIM and BID was observed in SW1271 cells than in H2171 cells (Fig. 3b). We have previously demonstrated that BID/BIM/ PUMA is required for $\mathrm{ABT}-737 / 263$ to induce apoptosis because ABT-737/263 displaces BID/BIM/PUMA from BCL-2/BCL- $\mathrm{X}_{\mathrm{L}}$ to activate $\mathrm{BAX} / \mathrm{BAK}$ indirectly ${ }^{5}$. Moreover, it was reported that BIM expression levels predict the sensitivity of cancer cells to 
a

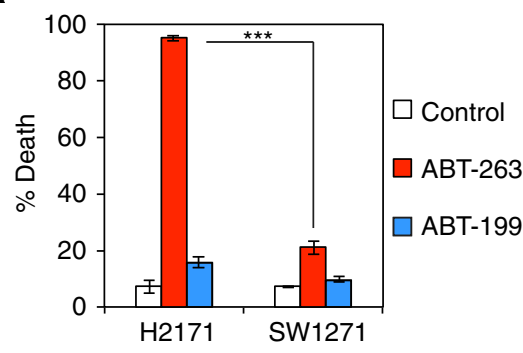

C

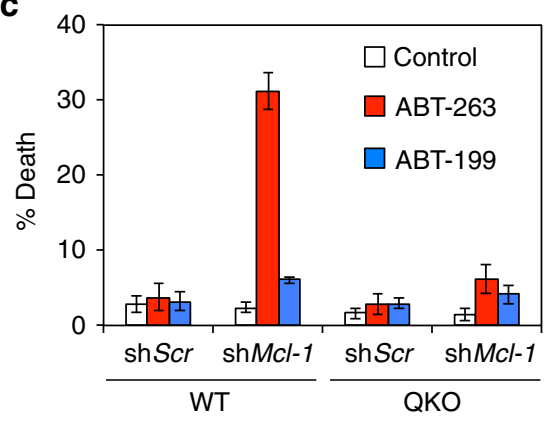

e

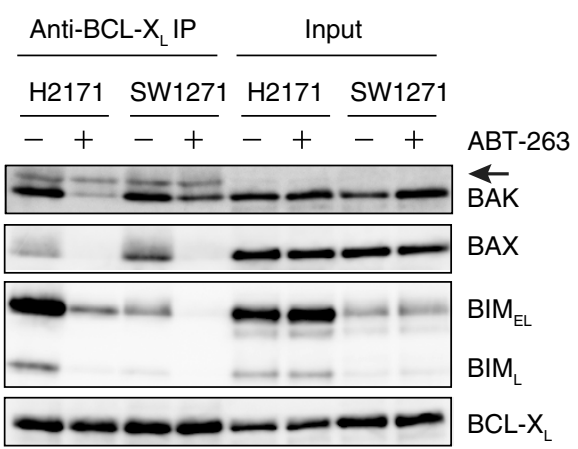

b

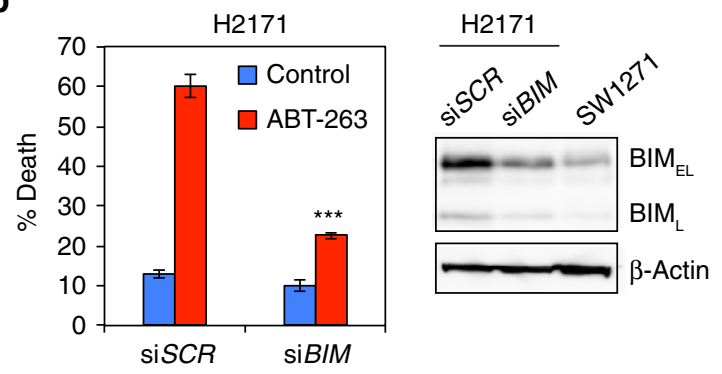

d
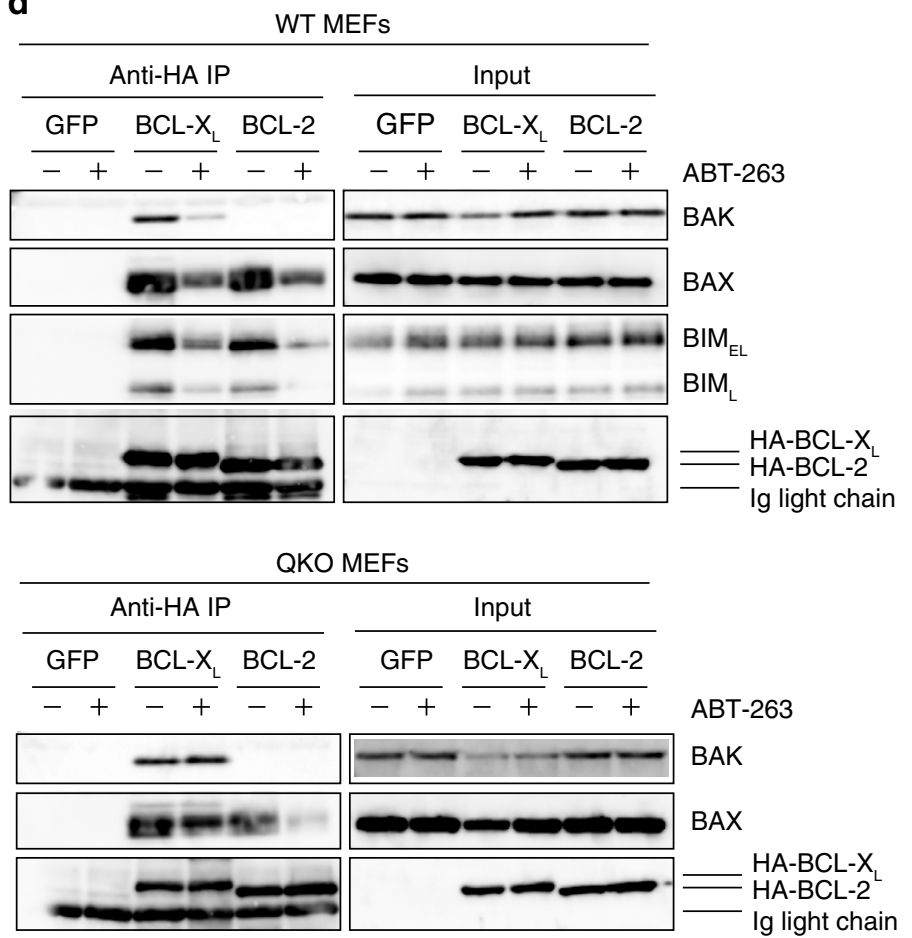

f
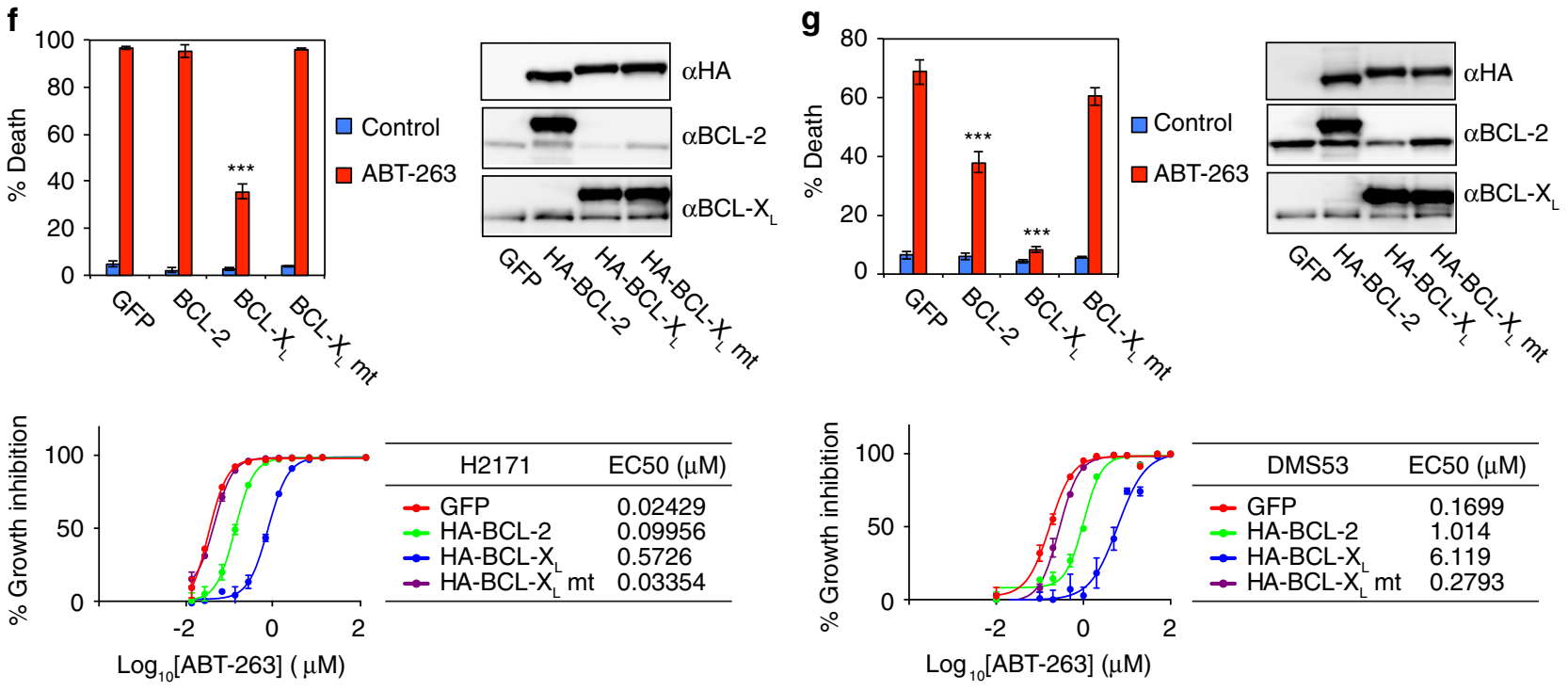
ABT-263 (ref. 25). To determine whether the differential sensitivities of these two cell lines to ABT-263 is caused by different BIM expression, BIM was silenced in H2171. Indeed, knockdown of BIM conferred resistance to ABT-263 in H2171 (Fig. 4b).

To further confirm that activator $\mathrm{BH} 3 \mathrm{~s}$ are required for $\mathrm{ABT}$ 263 to induce apoptosis, we employed activator BH3-deficient

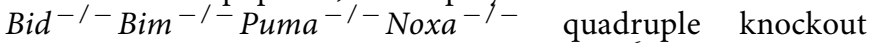
(QKO) mouse embryonic fibroblasts (MEFs) ${ }^{6}$. Knockdown of Mcl-1 sensitized wild-type MEFs to ABT-263- but not ABT199-induced apoptosis (Fig. 4c). However, ABT-263 failed to kill QKO cells with $\mathrm{Mcl}-1$ knockdown (Fig. 4c). This is in stark contrast to our reported knockdown experiments showing that concurrent $\mathrm{KD}$ of $\mathrm{Bcl}-2, \mathrm{Bcl}-\mathrm{x}_{\mathrm{L}}$ and $\mathrm{Mcl}-1$ is sufficient to induce autoactivation of BAX/BAK and apoptosis in QKO cells ${ }^{6}$. These data raise a testable hypothesis that $\mathrm{ABT}-263$ may be unable to disrupt the $\mathrm{BCL}-\mathrm{X}_{\mathrm{L}} / \mathrm{BAK}$ or $\mathrm{BCL}-\mathrm{X}_{\mathrm{L}} / \mathrm{BAX}$ interaction and thereby fails to kill $\mathrm{QKO}$ cells with $\mathrm{Mcl}-1$ knockdown. To examine this hypothesis, co-immunoprecipitation was performed. In wild-type MEFs, ABT-263 was able to reduce the interaction between $\mathrm{BCL}-\mathrm{X}_{\mathrm{L}}$ and $\mathrm{BAX}, \mathrm{BAK}$ or $\mathrm{BIM}$ (Fig. 4d). In contrast, ABT-263 failed to disrupt the interaction between $\mathrm{BCL}-\mathrm{X}_{\mathrm{L}}$ and $\mathrm{BAK}$ in $\mathrm{QKO}$ cells, whereas it partially reduced the interaction between $B C L-\mathrm{X}_{\mathrm{L}}$ and $\mathrm{BAX}$ (Fig. 4d). Of note, ABT-263 was able to reduce the interaction between BCL-2 and BAX or BIM in both wild-type and QKO cells (Fig. 4d). As reported ${ }^{6,34,35}$, BCL-2 only interacted with BAX but not BAK. Together, these data suggest that ABT-263 releases BIM and other activator $\mathrm{BH} 3$ s from BCL-2 and BCL- $\mathrm{X}_{\mathrm{L}}$ to disrupt the $\mathrm{BCL}-\mathrm{X}_{\mathrm{L}} / \mathrm{BAK}$ interaction indirectly. Accordingly, we examined whether ABT-263 has differential effects on disrupting the $\mathrm{BCL}-\mathrm{X}_{\mathrm{L}} / \mathrm{BAK}$ interaction in $\mathrm{H} 2171$ versus SW1271 cells. ABT-263 had minimal effect on the interaction between BCL-X $\mathrm{X}_{\mathrm{L}}$ and BAK in SW1271 with low BIM expression, whereas $\mathrm{ABT}-263$ greatly reduced this interaction in $\mathrm{H} 2171$ with high BIM expression (Fig. 4e). This is consistent with the notion that BIM is required to disrupt the $B C L-\mathrm{X}_{\mathrm{L}} /$ $\mathrm{BAK}$ interaction after its displacement from BCL- $\mathrm{X}_{\mathrm{L}}$ or BCL-2 by ABT-263. Collectively, ABT-263 is likely a poor $\mathrm{BCL}-\mathrm{X}_{\mathrm{L}}$ inhibitor in cells with low activator $\mathrm{BH} 3 \mathrm{~s}$ because it is unable to directly disrupt the BCL- $\mathrm{X}_{\mathrm{L}} / \mathrm{BAK}$ interaction.

Overexpression of $B C L-X_{L}$ confers resistance to ABT-263. We have reported that $\mathrm{BCL}-2, \mathrm{BCL}-\mathrm{X}_{\mathrm{L}}$ and $\mathrm{MCL}-1$ inhibit bimodal activation of $\mathrm{BAX} / \mathrm{BAK}$ by sequestering activator $\mathrm{BH} 3$ s and ' $\mathrm{BH} 3$ exposed' BAX/BAK monomers, respectively ${ }^{6}$. BCL- $\mathrm{X}_{\mathrm{L}}$ is superior to BCL-2 in preventing apoptosis, because BCL- $\mathrm{X}_{\mathrm{L}}$ but not BCL-2 can bind and inhibit $\mathrm{BAK}^{6}$. Given that ABT-263 could not directly disrupt the BCL- $\mathrm{X}_{\mathrm{L}} / \mathrm{BAK}$ interaction, we hypothesized that overexpression of $\mathrm{BCL}-\mathrm{X}_{\mathrm{L}}$ might provide more resistance to ABT-263 than overexpression of BCL-2 based on the unique capacity of BCL- $\mathrm{X}_{\mathrm{L}}$ to directly bind and inhibit 'BH3-exposed' BAK monomers. Indeed, overexpression of HA-tagged BCL- $\mathrm{X}_{\mathrm{L}}$ but not BCL-2 inhibited ABT-263-induced apoptosis in BCL- $\mathrm{X}_{\mathrm{L}^{-}}$ addicted $\mathrm{H} 2171$ (Fig. 4f). Notably, the BCL-X $\mathrm{L}_{\mathrm{L}} \mathrm{BH} 1$ mutant (G138E/R139L/I140N) that is unable to sequester proapoptotic BCL-2 members failed to protect $\mathrm{H} 2171$ (Fig. 4f). BCL-X overexpression resulted in a $\sim 24$-fold increase in the EC50 of ABT-263 whereas BCL-2 overexpression resulted in a $\sim 4$-fold increase in H2171 (Fig. 4f). Furthermore, overexpression of BCL$\mathrm{X}_{\mathrm{L}}$ in BCL-2-addicted DMS53 completely inhibited ABT-263induced apoptosis, whereas overexpression of BCL-2 only partially inhibited apoptosis (Fig. 4g). BCL-X $\mathrm{X}_{\mathrm{L}}$ overexpression resulted in a $\sim 36$-fold increase in the EC50 of ABT-263, whereas BCL-2 overexpression resulted in a $\sim 6$-fold increase in DMS53 (Fig. 4g). In both $\mathrm{H} 2171$ and DMS53, BCL-X $\mathrm{L}_{\mathrm{L}}$ overexpression increased the EC50 of ABT-263 by sixfold in comparison with BCL-2 overexpression. Overall, these results suggest that overexpression of $\mathrm{BCL}-\mathrm{X}_{\mathrm{L}}$ might be a potential resistance mechanism to ABT-263 due to the inability of ABT-263 to disrupt the $\mathrm{BCL}-\mathrm{X}_{\mathrm{L}} / \mathrm{BAK}$ interaction.

JQ1 neither downregulates $M C L-1$ nor cooperates with ABT-263. BRD4, a member of the BET family, is involved in the control of transcriptional elongation by $\mathrm{Pol}$ II through its recruitment of p-TEFb. Hence, we examined whether the BET inhibitor JQ1 (ref. 36) could recapitulate the effect of CDK9 inhibitors. JQ1 had no apparent single agent activity and failed to synergize with ABT-263 (Fig. 5a). Furthermore, there was no clear correlation of EC50s between dinaciclib and JQ1 (Fig. 5b), for example, DMS114 was sensitive to dinaciclib but not JQ1. Interestingly, three SCLC cell lines with $c-M Y C$ amplification, including H82, H2171 and H446, had the lowest EC50 to dinaciclib, which is consistent with a recent identification of CDK9 inhibition as a therapeutic strategy for c-MYC-overexpressing hepatocellular carcinoma ${ }^{37}$. c-MYC expression was indeed higher in $c$-MYC-amplified SCLC cell lines (Fig. 5c). As the EC50 was determined by growth inhibition, it might reflect anti-proliferative rather than death-inducing activity. Accordingly, cell death was quantified by annexin- $\mathrm{V}$ staining (Fig. 5d). Potent induction of apoptosis by dinaciclib at both $10 \mathrm{nM}$ (Fig. 2i) and $20 \mathrm{nM}$ (Fig. 5d) was observed in H82 and DMS114 cells that are addicted to MCL-1. In contrast, JQ1 failed to induce apoptosis in both H82 and DMS114 (Fig. 5d). JQ1 only induced marked apoptosis in $\mathrm{H} 446$ cells that are not addicted to any single anti-apoptotic member (Fig. 5d). Significant apoptosis was also observed in $\mathrm{H} 446$ cells treated with $20 \mathrm{nM}$ but not $10 \mathrm{nM}$ dinaciclib

\footnotetext{
Figure 4 | ABT-263 fails to disrupt the interaction between BCL- $\mathbf{X}_{\mathbf{L}}$ and BAK in the absence of activator BH3s. (a) H2171 and SW1271 cells were treated with vehicle, $1 \mu \mathrm{M}$ ABT-263 or $1 \mu \mathrm{M}$ ABT-199 for $48 \mathrm{~h}$. Cell death was quantified by annexin-V staining (mean $\pm \mathrm{s} . \mathrm{d} ., n=3$ ). (b) H2171 cells, transfected with scrambled siRNA (siSCR) or siRNA against BIM, were untreated or treated with ABT-263 for $24 \mathrm{~h}$. Cell death was quantified by annexin- $V$ staining (mean \pm s.d., $n=3$ ). The expression of BIM was assessed by an anti-BIM immunoblot. (c) SV40-transformed wild-type or Bid ${ }^{-/-B^{-}}{ }^{-/-P u m a}{ }^{-/}$

- Noxa ${ }^{-/-}$QKO MEFs, infected with retrovirus expressing shRNA against luciferase or Mcl-1, were untreated or treated with ABT-263 or ABT-199 for $24 \mathrm{~h}$. Cell death was quantified by annexin-V staining (mean \pm s.d., $n=3$ ). (d) SV40-transformed wild-type or Bid ${ }^{-/-}$Bim $^{-/-}$Puma $^{-/-}$Noxa $^{-/-}$QKO MEFs stably expressing GFP, HA-BCL- $\mathrm{X}_{\mathrm{L}}$ or HA-BCL-2, untreated or treated with ABT-263, were subjected to anti-HA immunoprecipitation. The input (5\%) and immunoprecipitates were assessed by immunoblot analysis. (e) H2171 and SW1271 cells, untreated or treated with ABT-263, were subjected to antiHA immunoprecipitation. The input (5\%) and immunoprecipitates were assessed by immunoblot analysis. Arrow indicates a cross-reactive band. (f) $\mathrm{H} 2171$ cells stably expressing GFP, HA-BCL-2, HA-BCL- $\mathrm{X}_{\mathrm{L}}$, or HA-BCL- $\mathrm{X}_{\mathrm{L}} \mathrm{BH} 1$ mutant were untreated or treated with $1 \mu \mathrm{M}$ ABT-263 for $24 \mathrm{~h}$. Cell death was quantified by annexin- $V$ staining (mean \pm s.d., $n=3$ ). EC50 of ABT-263 in H2171 cells was assessed by CellTiter-Glo assays at $48 \mathrm{~h}$. The expression of $\mathrm{HA}$-tagged $\mathrm{BCL}-2$ and $\mathrm{BCL}-\mathrm{X}_{\mathrm{L}}$ protein was assessed by immunoblot analysis. (g) DMS53 cells stably expressing GFP, HA-BCL-2, HA-BCL-X $\mathrm{X}_{\mathrm{L}}$ or HA-BCL-X $\mathrm{BH} 1$ mutant were untreated or treated with $1 \mu \mathrm{M} \mathrm{ABT}-263$ for $24 \mathrm{~h}$. Cell death was quantified by annexin-V staining (mean $\pm \mathrm{s}$.d., $n=3$ ). EC50 of $A B T-263$ in $\mathrm{H} 2171$ cells was assessed by CellTiter-Glo assays at $48 \mathrm{~h}$. The expression of HA-tagged BCL-2 and BCL- $\mathrm{X}_{\mathrm{L}}$ protein was assessed by immunoblot analysis. ${ }^{\star \star \star} P<0.001$ (Student's $t$-test). Unprocessed original scans of blots are shown in Supplementary Fig. 11.
} 

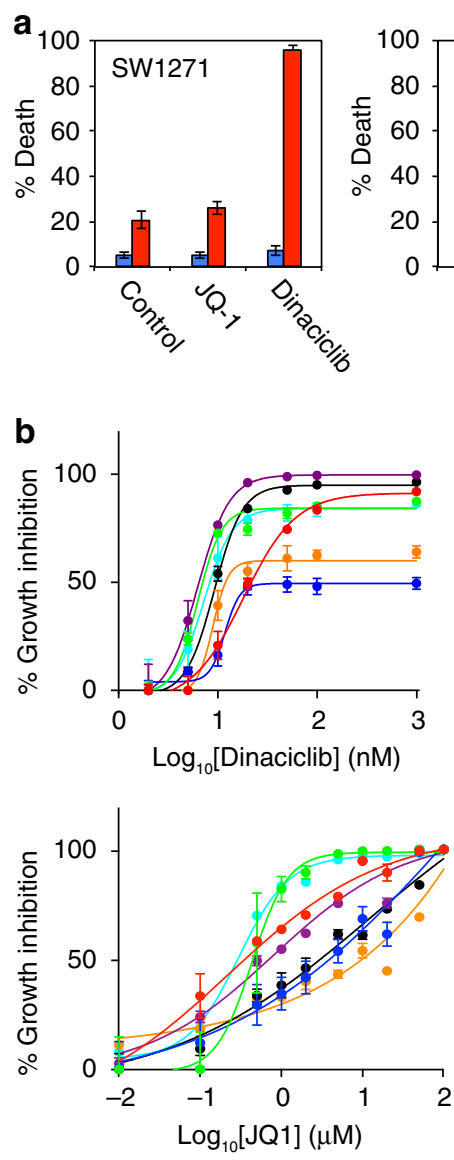

e
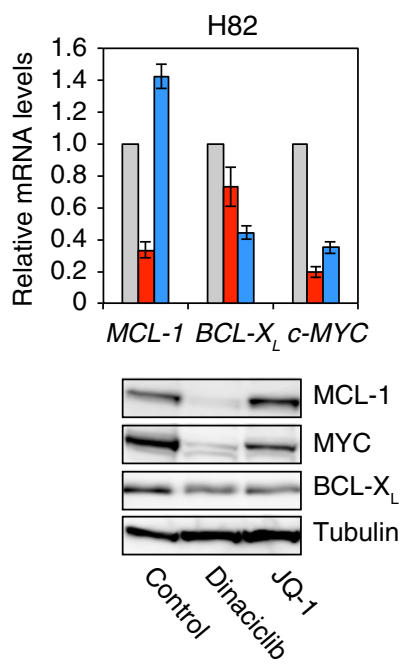
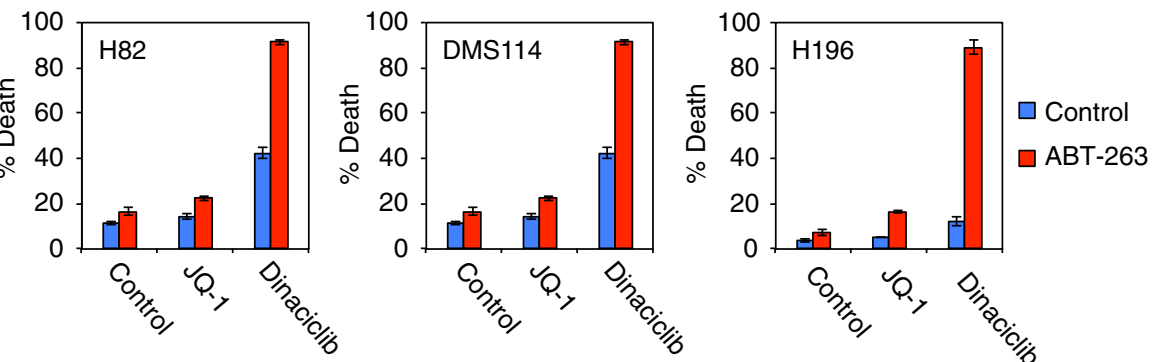

C
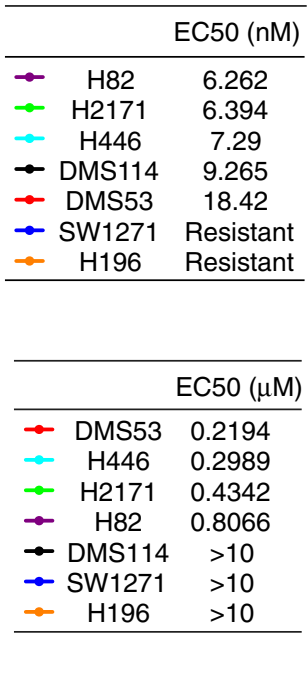

$\mathrm{H} 2171$
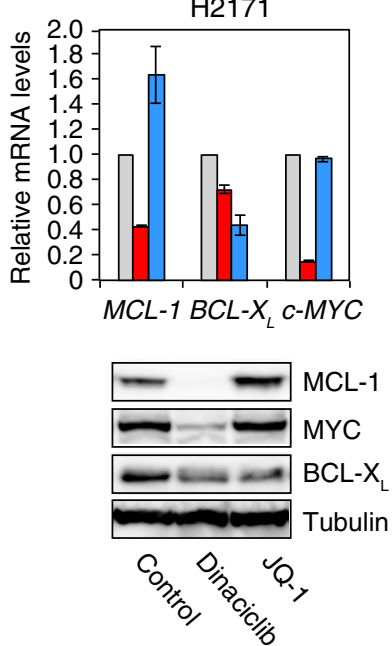
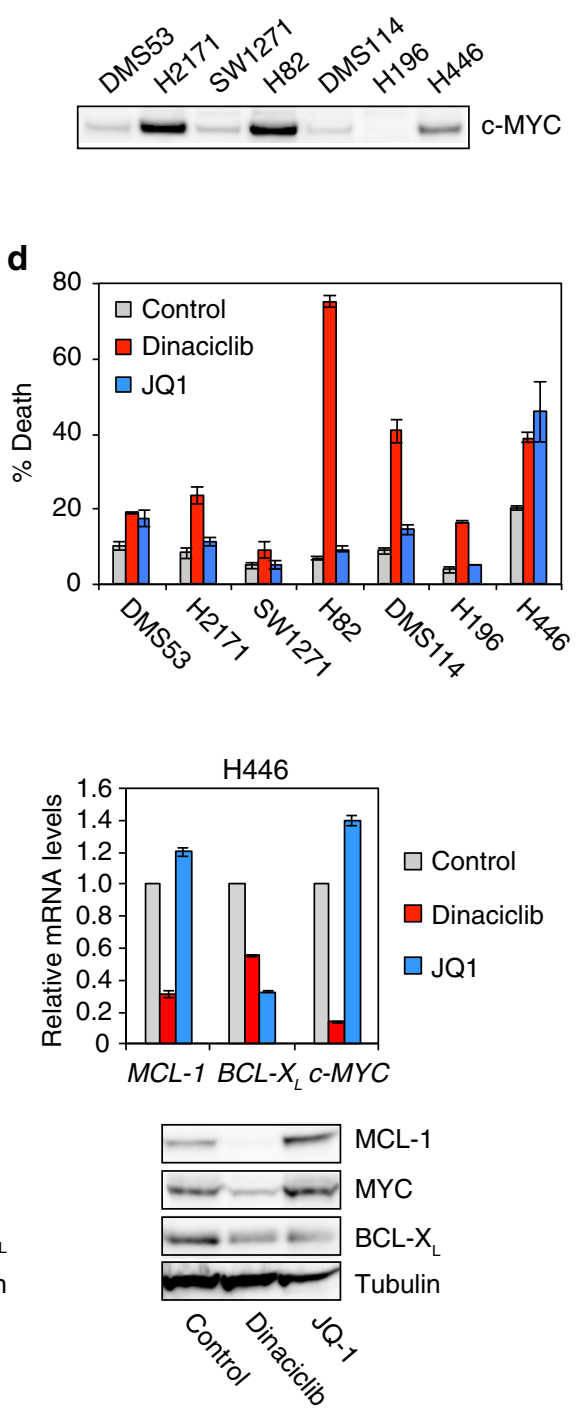

Figure 5 | Dinaciclib but not JQ1 synergizes with ABT-263 to kill SCLC cells. (a) The indicated SCLC cell lines were treated with vehicle, $1 \mu$ M JQ1, or dinaciclib (10 nM for H196 and H82, or $20 \mathrm{nM}$ for SW1271 and DMS114) in the absence or presence of $1 \mu \mathrm{M}$ ABT-263 for $24 \mathrm{~h}$. Cell death was quantified by

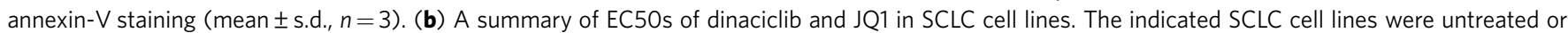
treated with increasing concentrations of dinaciclib or JQ1. Cell viability was assessed by CellTiter-Glo assays at $72 \mathrm{~h}$. (c) The expression of c-MYC in the indicated SCLC cell lines was assessed by an anti-c-Myc immunoblot. (d) The indicated SCLC cell lines were treated with vehicle, $1 \mu$ M JQ1, or 20 nM dinaciclib for $24 \mathrm{~h}$. Cell death was quantified by annexin- $\mathrm{V}$ staining (mean $\pm \mathrm{s} . \mathrm{d} ., \mathrm{n}=3$ ). (e) $\mathrm{H} 82, \mathrm{H} 2171$, and $\mathrm{H} 446 \mathrm{cells}$, treated with vehicle, $20 \mathrm{nM}$

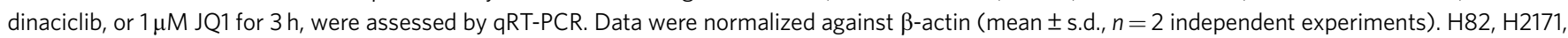
and $\mathrm{H} 446$ cells, treated with vehicle, dinaciclib, or JQ1 for $24 \mathrm{~h}$, were assessed by immunoblot analysis using the indicated antibodies. Unprocessed original scans of blots are shown in Supplementary Fig. 11.

(Figs $2 \mathrm{i}$ and $5 \mathrm{~d}$ ). Dinaciclib appeared to inhibit proliferation rather than inducing apoptosis in another $c$-MYC-amplified H2171 cells that are addicted to BCL- $\mathrm{X}_{\mathrm{L}}$ (Fig. 5b,d).
To this end, we envisioned that the reason why JQ1 fails to synergize with ABT-263 is most likely due to its inability to downregulate $M C L-1$. JQ1 was shown to prevent the binding of 
a

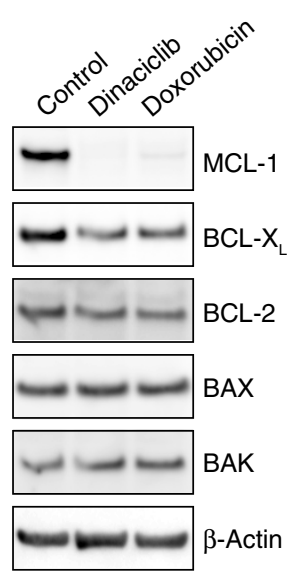

b

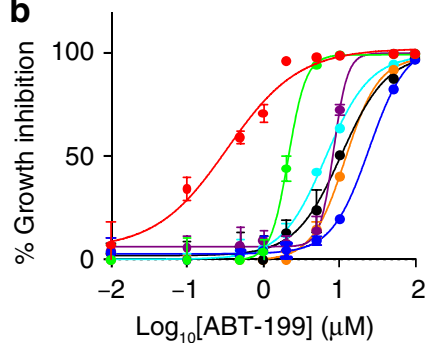

\begin{tabular}{|c|c|}
\hline & EC50 $(\mu \mathrm{M})$ \\
\hline - DMS53 & 0.386 \\
\hline$\Rightarrow \quad \mathrm{H} 2171$ & 2.127 \\
\hline H446 & 6.562 \\
\hline$\rightarrow \mathrm{H} 82$ & 8.347 \\
\hline - DMS114 & 10.520 \\
\hline$\rightarrow \mathrm{H} 196$ & 11.980 \\
\hline$\rightarrow$ SW1271 & 24.430 \\
\hline
\end{tabular}

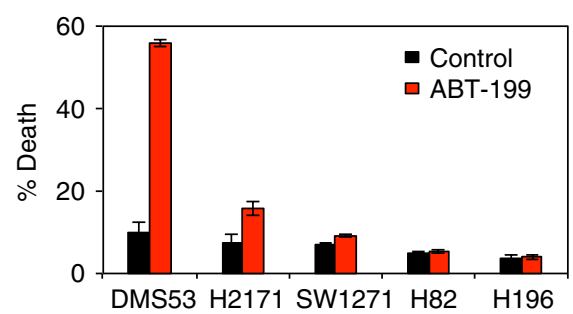

e

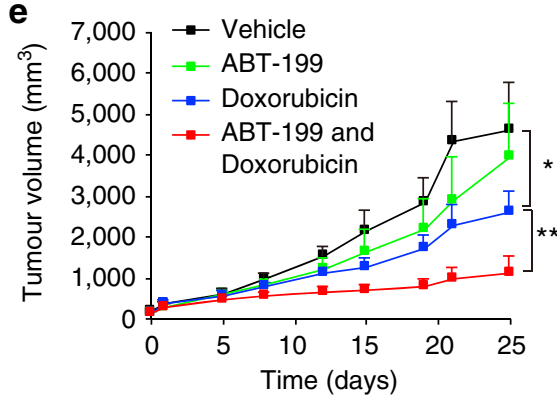

f

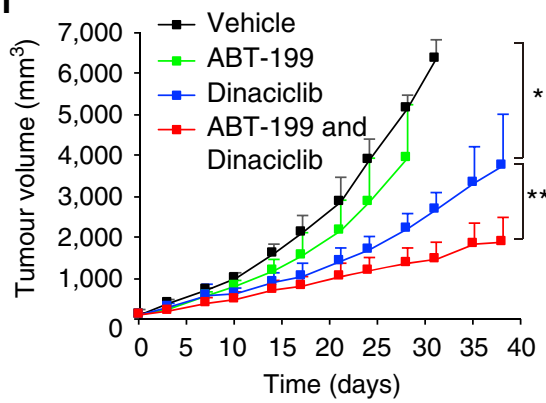

g
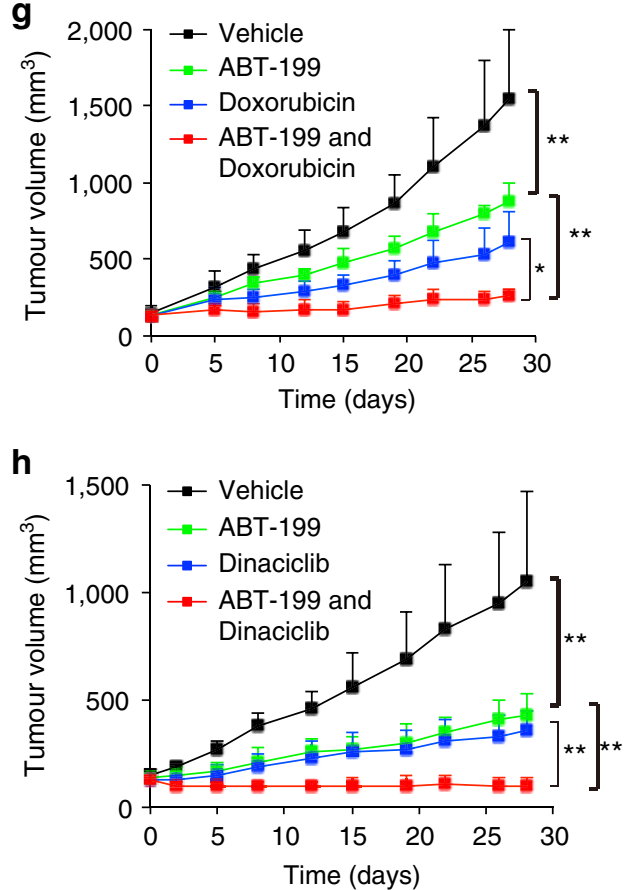
BRD4 to super-enhancers and cause transcription elongation defects that preferentially impact genes with super-enhancers, such as $c-M Y C$ and $B C L-X_{L}{ }^{38}$. We next examined the effect of dinaciclib and JQ1 on the expression of c-MYC, MCL-1 and BCL- $\mathrm{X}_{\mathrm{L}}$ in H82, H2171 and H446 cells (Fig. 5e). Dinaciclib greatly reduced the expression of both MCL-1 and c-MYC in these cell lines (Fig. 5e). In contrast, JQ1 induced MCL-1 (Fig. 5e), consistent with its inability to either kill MCL-1addicted cells or synergize with ABT-263. Intriguingly, c-MYC was reduced, unaffected or induced by JQ1 in these cell lines (Fig. 5e), which is similar to what has been reported for lung adenocarcinoma ${ }^{39}$. The anti-proliferative effect of JQ1 for these cell lines might be unrelated to c-MYC, as suggested for lung adenocarcinoma ${ }^{39}$. Collectively, our data suggest that dinaciclib will probably have single-agent anti-tumour activity against a subset of SCLC that either carry $c-M Y C$ amplification or are MCL-1-addicted.

Effective combination strategies for SCLC using ABT-199. Although doxorubicin and dinaciclib had more prompt and profound effects on reducing the expression of MCL-1 than BCL2 and BCL- $\mathrm{X}_{\mathrm{L}}$, we found that prolonged treatment of SCLC cells with these agents also partially reduced BCL- $\mathrm{X}_{\mathrm{L}}$ (Fig. 6a). These findings suggested a potentially effective therapeutic strategy in which combined ABT-199 with doxorubicin or dinaciclib might be sufficient to trigger apoptosis in SCLC. To test this idea, we first assessed EC50 of ABT-199 in various SCLC cell lines (Fig. 6b). Consistent with our characterization of DMS53 cells as BCL-2-addicted, DMS53 had the lowest EC50 to ABT-199 and was the only cell line that underwent potent apoptosis following ABT-199 treatment (Fig. 6b). Importantly, combined ABT-199 with doxorubicin or dinaciclib induced robust apoptosis in all tested SCLC cells including those ABT-263-resistant ones (Fig. 6c,d).

We next examined the in vivo anti-tumour efficacy of combined ABT-199 and doxorubicin or dinaciclib in the H446 xenograft model. Of note, H446 harbours mutations of RB1 and TP53 as well as $c-M Y C$ amplification, all of which are pathognomonic mutations of SCLC. Consistent with our in vitro study (Fig. 6c), monotherapy with ABT-199 or doxorubicin had minimal and modest effects on tumour growth, respectively (Fig. 6e). Importantly, combined ABT199 with doxorubicin markedly inhibited tumour growth without overt signs of toxicity (Fig. 6e). Similarly, combined ABT-199 with dinaciclib was superior to the respective monotherapy and greatly suppressed tumour growth (Fig. 6f). The therapeutic effects of combined ABT-199 with doxorubicin or dinaciclib were further demonstrated in a patient-derived SCLC xenograft (Fig. 6g,h). Collectively, these data suggest that combined ABT-199 with doxorubicin or dinaciclib could provide effective therapeutic strategies for SCLC.

\section{Discussion}

Evading cell death is one of the hallmarks of cancer ${ }^{40}$. Oncogenic transformation that drives uncontrolled cell-cycle progression often activates innate tumour-suppressive checkpoints that trigger apoptosis ${ }^{41}$. One plausible explanation is that BIM and PUMA are transcriptionally activated by E2F1 (ref. 42), a key cell cycle driver upon malignant transformation. To abrogate apoptotic checkpoints, cancer cells often overexpress antiapoptotic BCL-2 family proteins that sequester upregulated BIM and PUMA. Hence, many cancer cells are likely 'primed' to undergo apoptosis upon the administration of $\mathrm{BAD}$ and NOXA mimetics that displace BIM/PUMA from BCL-2/BCL- $\mathrm{X}_{\mathrm{L}}$ and MCL-1, respectively, to activate BAX/BAK ${ }^{12-14}$. As most normal cells do not have excessive BIM/PUMA readily complexed with anti-apoptotic BCL-2 members, they are less 'primed' and less susceptible to BAD or NOXA mimetics. In fact, clinical efficacy of ABT-263 (navitoclax) and ABT-199 (venetoclax) has been demonstrated in clinical trials ${ }^{12-14,18}$. In the era of precision medicine, it is paramount to identify and select patients who will respond to ABT-263 or ABT-199. Given that clinically applicable MCL-1 inhibitors are not yet available, strategies such as combination therapy to improve the therapeutic efficacy of ABT-263/199 are needed. Using protein expression ratios, we were able to predict the dependency of SCLC cell lines on BCL-2, BCL- $\mathrm{X}_{\mathrm{L}}$ or MCL-1 for survival. Moreover, we have identified two combination strategies through unbiased HTS, that is, combining ABT-263 with anthracyclines such as doxorubicin or with CDK9 inhibitors such as dinaciclib, which induced potent apoptosis in all examined SCLC cell lines regardless of their dependency on different anti-apoptotic BCL-2 members for survival (Fig. 7a). We further demonstrated that ABT-199 could replace ABT-263 for these two combination strategies, because both doxorubicin and dinaciclib also partially reduced BCL- $\mathrm{X}_{\mathrm{L}}$ (Fig. 7a). The combination strategies using ABT-199 are expected to reduce the toxicity associated with ABT-263.

Mechanistically, therapeutic modalities that either reduce MCL-1 mRNA/protein or induce proapoptotic BCL-2 family proteins could synergize with ABT-263 to induce apoptosis. Examples include anthracyclines and CDK9 inhibitors that downregulate $M C L-1$ (refs 26,30,31), histone deacetylase and MEK inhibitors that induce BIM $^{43-45}$, and phosphatidyl inositol 3-kinase inhibitors that induce PUMA ${ }^{44}$. Cancer type-specific synergizers of ABT-263 will probably prevail due to differing aberrant signalling pathways that coalesce into unique vulnerabilities. MEK and phosphatidyl inositol 3-kinase inhibitors were not identified in our HTS of SCLC in which kinase mutations are less common. Instead, anthracyclines and CDK9 inhibitors with transcriptional repressor function were identified as top hits in SCLC where near universal inactivation of p53 and RB1 directly impacts the transcriptional landscape.

\footnotetext{
Figure 6 | Doxorubicin or dinaciclib cooperates with ABT-199 to kill SCLC cells. (a) H196 cells, treated with dinaciclib or doxorubicin for $18 \mathrm{~h}$, were assessed by immunoblot analysis. (b) A summary of EC50s of ABT-199. Cell death response to $1 \mu \mathrm{M} \mathrm{ABT-199}$ was quantified by annexin- $V$ staining at $48 \mathrm{~h}$ (mean \pm s.d., $n=3$ ). (c) The indicated cell lines, treated with $2 \mu \mathrm{M}$ doxorubicin \pm 1 or $2 \mu \mathrm{M} \mathrm{ABT-199}$, were quantified by annexin- $V$ staining at $24 \mathrm{~h}$ for $\mathrm{H} 2171$ and $\mathrm{H} 446$ or at $48 \mathrm{~h}$ for $\mathrm{H} 196$ and SW1271 (mean \pm s.d., $n=3$ ). (d) The indicated cell lines, treated with $10-20 \mathrm{nM}$ dinaciclib \pm 1 or $2 \mu \mathrm{M} \mathrm{ABT-199}$, were quantified by annexin-V staining at $24 \mathrm{~h}$ for $\mathrm{H} 2171$ and $\mathrm{H} 446$, at $48 \mathrm{~h}$ for $\mathrm{H} 196$, or at $72 \mathrm{~h}$ for SW1271 (mean $\pm \mathrm{s} . \mathrm{d} ., n=3$ ). ${ }^{\star} P<0.05,{ }^{\star \star} P<0.01$ and ${ }^{\star \star \star} P<0.001$ (Student's $t$-test). (e) NSG mice bearing H446 xenografts were treated with vehicle $(n=6)$, ABT-199 $\left(100 \mathrm{mg} \mathrm{kg}{ }^{-1}, n=8\right)$, doxorubicin $\left(2 \mathrm{mg} \mathrm{kg}^{-1}, n=8\right)$, or combined ABT-199 and doxorubicin $(n=8)$. tumour volumes were measured twice weekly by caliper (mean $\pm \mathrm{s} . \mathrm{d}$.). ${ }^{\star} P=0.0022$ and ${ }^{\star \star} P<0.0001$ (two-way analysis of variance (ANOVA)). (f) NSG mice bearing H446 xenografts were treated with vehicle $(n=8)$, ABT-199 $\left(100 \mathrm{mg} \mathrm{kg}^{-1}\right.$, $n=8$ ), dinaciclib (20 mg kg $\left.{ }^{-1}, n=8\right)$, or combined ABT-199 and dinaciclib $(n=8) .{ }^{\star} P=0.0002 ;{ }^{\star \star} P=0.0034$ (two-way ANOVA). (g) NSG mice bearing patient-derived SCLC xenografts were treated with vehicle $(n=5)$, ABT-199 (100 mg kg $\left.{ }^{-1}, n=4\right)$, doxorubicin $\left(2 \mathrm{mg} \mathrm{kg}^{-1}, n=6\right)$, or combined ABT-199 and doxorubicin $(n=4) .{ }^{\star} P=0.0029 ;{ }^{\star}{ }^{*} P<0.0001$ (two-way ANOVA). (h) NSG mice bearing patient-derived SCLC xenografts were treated with vehicle $(n=6)$, ABT-199 (100 mg kg-1, $n=6)$, dinaciclib (30 $\left.\mathrm{mg} \mathrm{kg}^{-1}, n=6\right)$, or combined ABT-199 and dinaciclib $(n=8)$. ${ }^{\star \star} P<0.0001$ (two-way ANOVA). Unprocessed original scans of blots are shown in Supplementary Fig. 11.
} 

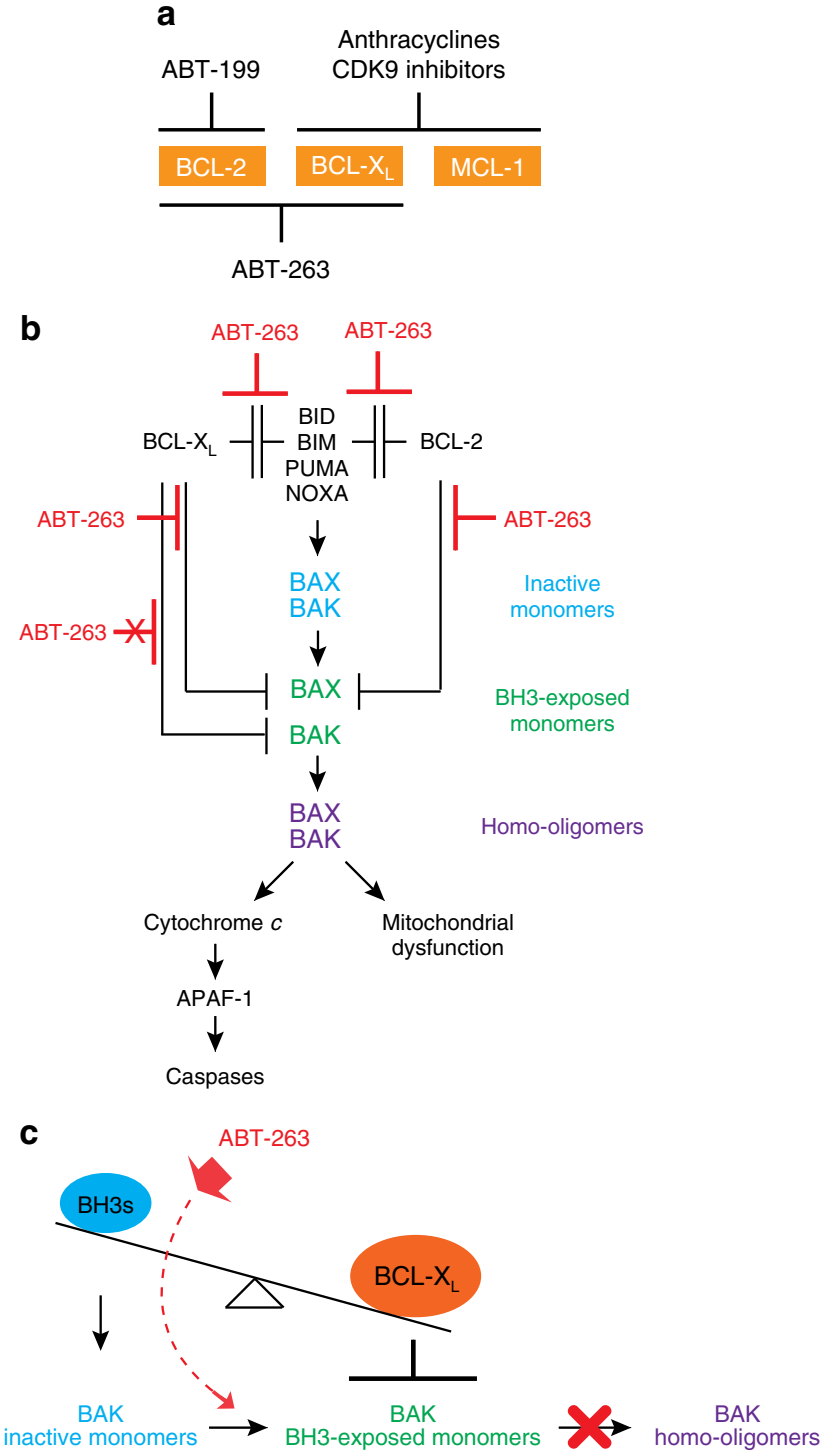

Figure 7 | Schematic depiction of mechanism-guided targeting of the BCL-2 family for cancer therapy. (a) Differential targeted inhibition of BCL2, BCL-X or MCL-1 by ABT-199, ABT-263, anthracyclines, or CDK9 inhibitors. (b) A schematic depicts the interconnected hierarchical model of cell death regulation by the $B C L-2$ family proteins and differential inhibitory activity of $A B T-263$ against $B C L-2$ versus $B C L-X_{L}$. ABT-263 prevents $B C L-2$ and $B C L-X_{L}$ from sequestering both activator $B H 3 s$ and $B A X$. In contrast, $A B T-263$ fails to prevent $B C L-X_{L}$ from sequestering BAK. (c) BCL- $X_{L}$ overabundance confers resistance to $A B T-263$ where $B C L-X_{L}$ prevents the 'BH3-exposed' BAK monomers from undergoing homo-oligomerization.

It was reported that oncogenic and lineage-specific transcription factors are associated with super-enhancers in SCLC ${ }^{46}$, and that JQ1 preferentially inhibits the transcription of genes with super-enhancers ${ }^{38}$, suggesting that JQ1 may provide a therapeutic effect for SCLC. However, JQ1 neither downregulated MCL-1 nor sensitized SCLC cell lines to ABT-263 and the EC50s of JQ1 did not correlate with those of dinaciclib in the examined SCLC cell lines. Interestingly, we found that SCLC cell lines with $c-M Y C$ amplification were more sensitive to dinaciclib and dinaciclib alone was sufficient to induce apoptosis in MCL-1-addicted SCLC cell lines. Together, our data suggest that CDK9 inhibitors may have single-agent anti-tumour activity against a subset of SCLC that either carry $c-M Y C$ amplification or are MCL-1-addicted. It was reported that SCLC is highly sensitive to a covalent CDK7 inhibitor, THZ1 (ref. 46). During transcription initiation, the carboxy-terminal domain of Pol II is phosphorylated on Ser5 by the TFIIH-associated kinase CDK7, and as Pol II elongates, Ser2 is increasingly phosphorylated by $\mathrm{CDK} 9 / \mathrm{p}-\mathrm{TEFb}$, while Ser5 phosphorylation is gradually removed by phosphatases ${ }^{29}$. In fact, THZ1 was shown to reduce the phosphorylation of both Ser5 and Ser2 in SCLC ${ }^{46}$. It is possible that downregulation of MCL-1 is responsible for the death-inducing activity of THZ1 in SCLC.

Using RNAi to determine the dependency on individual antiapoptotic BCL-2 members for survival, we have classified SCLC cell lines into BCL-2-addicted, BCL- $\mathrm{X}_{\mathrm{L}}$-addicted, MCL-1addicted or non-addicted to any single anti-apoptotic BCL-2 member. As expected, the BCL-2-addicted SCLC cells were sensitive to both ABT-199 and ABT-263, whereas the MCL-1addicted SCLC cells were insensitive to both. Surprisingly, the two BCL- $\mathrm{X}_{\mathrm{L}}$-addicted SCLC cell lines showed differential sensitivity to ABT-263 due to different BIM expression. We have previously established an interconnected hierarchical model that explains how the intricate interplays between three BCL-2 subfamilies dictate cellular survival versus death ${ }^{6}$ (Fig. 7b). Antiapoptotic BCL-2, BCL- $\mathrm{X}_{\mathrm{L}}$ and MCL-1 inhibit apoptosis through sequestering activator $\mathrm{BH} 3$ s from activating $\mathrm{BAX} / \mathrm{BAK}$, providing a frontline protection against apoptotic insults ${ }^{2-4}$. In addition, BCL-2, BCL- $\mathrm{X}_{\mathrm{L}}$ and MCL-1 can sequester partially activated, $\mathrm{BH} 3$-exposed, $\mathrm{BAX} / \mathrm{BAK}$ monomers to prevent the homooligomerization of BAX/BAK (ref. 6), serving as a fail-safe mechanism or the second line of defense. We showed that ABT263 prevented BCL- 2 and $B C L-X_{L}$ from sequestering BIM and $\mathrm{BAX}$, but failed to directly disrupt the interaction of BCL- $\mathrm{X}_{\mathrm{L}}$ and BAK (Figs 4 and $7 \mathrm{~b}$ ). The inability of ABT-263 to breach the second-line defense of $\mathrm{BCL}-\mathrm{X}_{\mathrm{L}}$ against $\mathrm{BAK}$ foretells major limitations to its use. ABT-263 is probably ineffective in treating $\mathrm{BCL}-\mathrm{X}_{\mathrm{L}}$-addicted cancers with low expression of activator $\mathrm{BH} 3 \mathrm{~s}$ such as BIM, as well as cancers with $\mathrm{BCL}-\mathrm{X}_{\mathrm{L}}$ overabundance, which prevents the $\mathrm{BH} 3$-exposed $\mathrm{BAK}$ monomers from undergoing homo-oligomerization (Fig. 7c). It is intriguing that ABT-263 fails to directly dissociate the BCL- $\mathrm{X}_{\mathrm{L}} / \mathrm{BAK}$ complexes given the high binding affinity of ABT-263 to the hydrophobic dimerization groove of $\mathrm{BCL}-\mathrm{X}_{\mathrm{L}}$. $\mathrm{BAX}$ appears to differ from $\mathrm{BAK}$ because ABT-263 could disrupt the interaction between BAX and BCL- $\mathrm{X}_{\mathrm{L}}$ or BCL-2 (Figs 4 and $7 \mathrm{~b}$ ). It is possible that additional contact sites between BCL- $\mathrm{X}_{\mathrm{L}}$ and $\mathrm{BAK}$ further strengthen the $\mathrm{BH} 3$-in-groove heterodimers and/or prevent the accessibility of ABT-263 to the dimerization pocket. Alternatively, the lipid milieu of $\mathrm{MOM}$ where $\mathrm{BCL}-\mathrm{X}_{\mathrm{L}}$ and $\mathrm{BAK}$ reside may modulate their interaction. Nonetheless, targeting the interaction between BCL- $\mathrm{X}_{\mathrm{L}}$ and BAK is essential for the treatment of 'poorly primed' yet BCL- $\mathrm{X}_{\mathrm{L}}$-addicted cancer (Fig. $7 \mathrm{c}$ ), which remains unaddressed by ABT-263.

A potential limitation for our prediction model extends to cancers with high expression of BCL2A1 or BCL-W. Notably, SCLC has the lowest expression of BCL2A1 among the 1,000 cell lines analysed by the Cancer Cell Line Encyclopedia project ${ }^{47}$. The predominant association of a specific anti-apoptotic BCL-2 member with BIM and BAK has been shown to predict dependency for survival in multiple myeloma and AML, respectively ${ }^{48,49}$. Thus far, most of the prediction methods have been focused on the identification of ABT-263-responsive cancers. BH3 profiling is one such example, which evaluates apoptotic sensitivity or 'mitochondrial priming' of cancer cells by measuring whether $\mathrm{BH} 3$ domain peptides induce $\mathrm{MOMP}^{50}$. Of note, our prediction method may not be applicable to highly primed cancer such as hematological malignancies that highly express BIM as revealed by the Cancer Cell Line Encyclopedia project. Highly primed cancers may be sensitive to the 
knockdown of any individual anti-apoptotic BCL-2 member even though they predominantly express one anti-apoptotic BCL-2 member. Accordingly, highly primed cancers may be sensitive to ABT-263 even though they are MCL-1-dependent, which has been shown in multiple myeloma ${ }^{48}$.

Our study establishes a framework for identifying and targeting the differential addiction to anti-apoptotic BCL-2 family proteins in SCLC, which could potentially be applied to other cancer types. Combined inhibition of BCL-2, BCL- $\mathrm{X}_{\mathrm{L}}$ and MCL-1 through mechanism-guided combination therapy will provide the most effective and a potentially universal therapeutic strategy to eradicate cancer cells through apoptotic induction. We presented two such strategies, that is, the combinations of ABT-199 and doxorubicin or dinaciclib. Furthermore, we have identified the inability of ABT-263 to prevent BCL- $\mathrm{X}_{\mathrm{L}}$ from sequestering BAK as a major limitation of its application in cancer therapy. A better understanding of the BCL- $\mathrm{X}_{\mathrm{L}} / \mathrm{BAK}$ interaction may aid in the development of a better BCL- $\mathrm{X}_{\mathrm{L}}$ inhibitor, which is critical for the advancement of targeted therapies against BCL-2 family, as is the development of a clinically applicable MCL-1 inhibitor. Overall, this study demonstrates the feasibility of exploiting the interconnected hierarchical cell death model for the development of specific therapeutic strategies aimed at direct activation of mitochondrial apoptosis in susceptible cancer cells.

\section{Methods}

Cell culture and viability assay. NCI-H196, SW1271, NCI-H2171, NCI-H446, DMS53 and DMS114 were obtained from the American Type Culture Collection. H82 was obtained from Dr Charles Rudin at Memorial Sloan Kettering Cancer Center. All cell lines were cultured according to the recommendations of the American Type Culture Collection. SV40-transformed wild-type or Bid Bim $^{-1-P_{m m a}}{ }^{-1-}$ Noxa $^{-1-}$ MEFs were described previously ${ }^{6}$. Cell death was quantified by Annexin V (BioVison) staining, followed by flow cytometric analyses using an LSRFortessa (BD Biosciences). Data were analysed using FACSDiva (BD Biosciences). For $\mathrm{EC}_{50}$ determination, cell viability was assessed by the CellTiterGlo luminescence assays (Promega) using 96-well plates and a luminescent plate reader (SpectraMax M2e, Molecular Devices). $\mathrm{EC}_{50}$ value was calculated using Prism software (GraphPad). P-values for statistical analyses were obtained with Student's $t$-test. The chemicals used are as follows: ABT-737 (Selleck Chemicals); ABT-263 (AbbVie and Selleck Chemicals); ABT-199 (AbbVie and Selleck Chemicals); dinaciclib (Selleck Chemicals); SNS-032 (Selleck Chemicals); AZD5438 (Selleck Chemicals); JQ1 (Selleck Chemicals); doxorubicin (Sigma); etoposide (Sigma); cisplatin (Sigma); camptothecin (Sigma); rapamycin (Sigma); everolimus (Selleck Chemicals); AZD8580 (Selleck Chemicals); FDA-panel for HTS (Sigma); actinomycin D (Sigma); PHA-793887 (Selleck Chemicals); and pathway inhibitors for HTS (Selleck Chemicals).

High-throughput screening. HTS was performed by the HTS Core Facility at Memorial Sloan Kettering Cancer Center using an automated 384-well platform. H196 cells were screened against FDA-approved anti-cancer agents (Sigma) and a pathway inhibitor library (Selleck Chemicals) in combination with ABT-737. For the screening of FDA panel, H196 cells were treated with each compound at 12 twofold serial dilution doses starting from $10 \mu \mathrm{M}$ in the absence or presence of $1 \mu \mathrm{M}$ ABT-737. Compounds were screened in duplicate and the growth inhibition was assessed by Alamar Blue assays at 3 days post drug treatment. The average Z' factor for the assay robustness was 0.45 . Dose response curves for the FDA panel were fitted using logistic four-parameter sigmoid regression equations and EC50 values were calculated by Sigma-plot (Systat Software). For the screening of pathway inhibitors, H196 cells were treated with each compound at $2 \mu \mathrm{M}$ in the absence or presence of $1 \mu \mathrm{M}$ ABT-737. Compounds were screened in duplicate and the growth inhibition was assessed by Alamar Blue assays at 3 days post drug treatment. The average $Z$ ' factor for the assay robustness was 0.48 . Scatterplot analysis of the average percentage growth inhibition was performed to identify compounds that synergized with ABT-737 to kill H196 cells.

RNA interference. siRNA oligos were purchased from Ambion Silencer Select oligos (Applied Biosystems), the sequences of which are summarized in Supplementary Data 3. siRNA oligos were reverse transfected with Lipofectamine RNAiMAX (Invitrogen) to a final concentration of $10 \mathrm{nM}$.

Reverse transcription and quantitative PCR. Total RNA was extracted from cells with TRIZOL (Life Technologies) according to the manufacturer's instruction. Reverse transcription was performed with oligo-dT plus random decamer primers
(Ambion) with SuperScript II (Life Technologies). The primers are summarized in Supplementary Data 3. Quantitative PCR was performed with SYBR Green Master Mix (Applied Biosystems) in duplicate with the indicated gene-specific primers Quantitative PCR was performed on an ABI Prism 7,300 sequence detection system (Applied Biosystems). Data were analysed by normalization against $\beta$-actin.

Immunoblot analysis and immunoprecipitation. Cells were lysed in RIPA buffer. Protein concentration was determined by BCA kit (Pierce). Twenty-five to $50 \mu \mathrm{g}$ of proteins were resolved by $10 \%$ NuPAGE (Life Technologies), transferred onto polyvinylidene difluoride membrane (Immobilon-P, Millipore). Antibody detection was accomplished using enhanced chemiluminescence method (Western Lightning, PerkinElmer) and LAS-3000 Imaging system (FUJFILM). Antibodies used for immunoblot analysis are listed as followed: anti-BAK (NT, Millipore), antiBAX (N-20, Santa Cruz Biotechnology), anti-BIM (C34C5, Cell Signaling Technology), anti-PUMA (no. 4976, Cell Signaling Technology), anti-NOXA (ab13654, Abcam), anti-BID (FL-195, Santa Cruz Biotechnology), anti-BCL-2 (6C8), antiBCL-X $\mathrm{L}_{\mathrm{L}}$ (7B2.5), anti-MCL-1 (S-19, Santa Cruz Biotechnology), anti-phospho RNA Pol II S2 (A300-654A, Betyl Laboratories), anti-CDK9 (C-20, Santa Cruz Biotechnology), anti-MYC (06-340, Millipore), anti- $\alpha$-Tubulin (T6199, Sigma) and anti- $\beta$-actin (A-1978, Chemicon). To quantify protein expression ratios, immunoblots were assessed by ImageJ (v. 1.46r). The relative expression ratios of BCL-2, BCL- $\mathrm{X}_{\mathrm{L}}$, or MCL-1 to combined BCL-2, BCL- $\mathrm{X}_{\mathrm{L}}$ and MCL-1 were calculated using the quantified intensity of each protein normalized against $\alpha$-Tubulin. Co-immunoprecipitation was performed as described ${ }^{6}$ using the anti-HA (12CA5) or antiBCL- $\mathrm{X}_{\mathrm{L}}$ (7B2.5) antibodies and analysed by $10 \%$ NuPAGE (Life Technologies) and immunoblots.

Xenograft studies. Animal experiments were performed in accordance with the MSKCC Institutional Animal Care and Use Committee. Sex-matched 7-8 week-old NOD/SCID/IL2R $\gamma^{\text {null }}$ (NSG) mice (Jackson Laboratories) were injected subcutaneously with $3 \times 10^{6} \mathrm{H} 446$ cells in $0.2 \mathrm{ml} 50 \%$ Matrigel (BD Biosciences). The PDX model (ECLC9) was derived from a patient previously treated with carboplatin and etoposide in accordance with the MSKCC Institutional Review Board approved tissue collection protocol with informed consent from the patient and propagated in NSG mice. Tumour growth was monitored twice weekly by calipers (volume $=$ length $\times$ width $^{2} / 2$ ). When tumours reached $\sim 150 \mathrm{~mm}^{3}$, tumour-bearing mice were randomized according to tumour volume. ABT-199 $\left(100 \mathrm{mg} \mathrm{kg}^{-1}\right)$ was administered by oral gavage at a weekly schedule of 5 days on and 2 days off. ABT-199 was formulated in $10 \%$ ethanol, $30 \%$ polyethylene glycol 400 and $60 \%$ Phosal 50 PG. Doxorubicin was formulated in PBS and administered intravenously once weekly at $2 \mathrm{mg} \mathrm{kg}^{-1}$. Dinaciclib was formulated in $20 \%$ hydroxypropyl $\beta$ cyclodextrin and administered intraperitoneally twice weekly at 20 or $30 \mathrm{mg} \mathrm{kg}^{-1}$ $P$-values for statistical analyses were obtained with two-way analysis of variance.

Data availability. All relevant data not presented in the main figures or Supplementary Information is available from the corresponding author upon request.

\section{References}

1. Czabotar, P. E., Lessene, G., Strasser, A. \& Adams, J. M. Control of apoptosis by the BCL-2 protein family: implications for physiology and therapy. Nat. Rev. Mol. Cell Biol. 15, 49-63 (2014).

2. Cheng, E. H. et al. BCL-2, BCL- $\mathrm{X}_{\mathrm{L}}$ sequester BH3 domain-only molecules preventing BAX- and BAK-mediated mitochondrial apoptosis. Mol. Cell 8, 705-711 (2001).

3. Kim, H. et al. Hierarchical regulation of mitochondrion-dependent apoptosis by BCL-2 subfamilies. Nat. Cell Biol. 8, 1348-1358 (2006).

4. Kim, H. et al. Stepwise activation of BAX and BAK by tBID, BIM, and PUMA initiates mitochondrial apoptosis. Mol. Cell 36, 487-499 (2009).

5. Ren, D. et al. BID, BIM, and PUMA are essential for activation of the BAX- and BAK-dependent cell death program. Science 330, 1390-1393 (2010).

6. Chen, H. C. et al. An interconnected hierarchical model of cell death regulation by the BCL-2 family. Nat. Cell Biol. 17, 1270-1281 (2015).

7. Dewson, G. et al. To trigger apoptosis, Bak exposes its $\mathrm{BH} 3$ domain and homodimerizes via BH3:groove interactions. Mol. Cell 30, 369-380 (2008).

8. Willis, S. N. et al. Apoptosis initiated when BH3 ligands engage multiple Bcl-2 homologs, not Bax or Bak. Science 315, 856-859 (2007).

9. Korsmeyer, S. J. Bcl-2 initiates a new category of oncogenes: regulators of cell death. Blood 80, 879-886 (1992).

10. Oltersdorf, T. et al. An inhibitor of Bcl-2 family proteins induces regression of solid tumours. Nature 435, 677-681 (2005).

11. Tse, C. et al. ABT-263: a potent and orally bioavailable Bcl-2 family inhibitor. Cancer Res. 68, 3421-3428 (2008).

12. Davids, M. S. \& Letai, A. Targeting the B-cell lymphoma/leukemia 2 family in cancer. J. Clin. Oncol. 30, 3127-3135 (2012).

13. Walensky, L. D. From mitochondrial biology to magic bullet: navitoclax disarms BCL-2 in chronic lymphocytic leukemia. J. Clin. Oncol. 30, 554-557 (2012). 
14. Anderson, M. A., Huang, D. \& Roberts, A. Targeting BCL2 for the treatment of lymphoid malignancies. Semin. Hematol. 51, 219-227 (2014).

15. Gandhi, L. et al. Phase I study of navitoclax (ABT-263), a novel Bcl-2 family inhibitor, in patients with small-cell lung cancer and other solid tumors. J. Clin. Oncol. 29, 909-916 (2011).

16. Rudin, C. M. et al. Phase II study of single-agent navitoclax (ABT-263) and biomarker correlates in patients with relapsed small cell lung cancer. Clin. Cancer Res. 18, 3163-3169 (2012).

17. Souers, A. J. et al. ABT-199, a potent and selective BCL-2 inhibitor, achieves antitumor activity while sparing platelets. Nat. Med. 19, 202-208 (2013).

18. Roberts, A. W. et al. Targeting BCL2 with venetoclax in relapsed chronic lymphocytic leukemia. N. Engl. J. Med. 374, 311-322 (2016).

19. Leverson, J. D. et al. Exploiting selective BCL-2 family inhibitors to dissect cell survival dependencies and define improved strategies for cancer therapy. Sci. Transl. Med. 7, 279 ra240 (2015).

20. Semenova, E. A., Nagel, R. \& Berns, A. Origins, genetic landscape, and emerging therapies of small cell lung cancer. Genes Dev. 29, 1447-1462 (2015).

21. William, Jr W. N. \& Glisson, B. S. Novel strategies for the treatment of small-cell lung carcinoma. Nat. Rev. Clin. Oncol. 8, 611-619 (2011).

22. George, J. et al. Comprehensive genomic profiles of small cell lung cancer. Nature 524, 47-53 (2015)

23. Peifer, M. et al. Integrative genome analyses identify key somatic driver mutations of small-cell lung cancer. Nat. Genet. 44, 1104-1110 (2012).

24. Shoemaker, A. R. et al. Activity of the Bcl-2 family inhibitor ABT-263 in a panel of small cell lung cancer xenograft models. Clin. Cancer Res. 14, 3268-3277 (2008).

25. Faber, A. C. et al. Assessment of ABT-263 activity across a cancer cell line collection leads to a potent combination therapy for small-cell lung cancer. Proc. Natl Acad. Sci. USA 112, E1288-E1296 (2015).

26. Wei, G. et al. Chemical genomics identifies small-molecule MCL1 repressors and BCL-xL as a predictor of MCL1 dependency. Cancer Cell 21, 547-562 (2012).

27. Brasca, M. G. et al. Optimization of 6,6-dimethyl pyrrolo[3,4-c]pyrazoles: Identification of PHA-793887, a potent CDK inhibitor suitable for intravenous dosing. Bioorg. Med. Chem. 18, 1844-1853 (2010).

28. Wang, S. \& Fischer, P. M. Cyclin-dependent kinase 9: a key transcriptional regulator and potential drug target in oncology, virology and cardiology. Trends Pharmacol. Sci. 29, 302-313 (2008).

29. Hsin, J. P. \& Manley, J. L. The RNA polymerase II CTD coordinates transcription and RNA processing. Genes Dev. 26, 2119-2137 (2012).

30. MacCallum, D. E. et al. Seliciclib (CYC202, R-Roscovitine) induces cell death in multiple myeloma cells by inhibition of RNA polymerase II-dependent transcription and down-regulation of Mcl-1. Cancer Res. 65, 5399-5407 (2005).

31. Shapiro, G. I. Cyclin-dependent kinase pathways as targets for cancer treatment. J. Clin. Oncol. 24, 1770-1783 (2006).

32. Kumar, S. K. et al. Dinaciclib, a novel CDK inhibitor, demonstrates encouraging single-agent activity in patients with relapsed multiple myeloma. Blood 125, 443-448 (2015).

33. Gardner, E. E. et al. Rapamycin rescues ABT-737 efficacy in small cell lung cancer. Cancer Res. 74, 2846-2856 (2014).

34. Cheng, E. H., Sheiko, T. V., Fisher, J. K., Craigen, W. J. \& Korsmeyer, S. J. VDAC2 inhibits BAK activation and mitochondrial apoptosis. Science 301, 513-517 (2003).

35. Willis, S. N. et al. Proapoptotic Bak is sequestered by Mcl-1 and Bcl- $\mathrm{x}_{\mathrm{L}}$, but not Bcl-2, until displaced by BH3-only proteins. Genes Dev. 19, 1294-1305 (2005).

36. Filippakopoulos, P. et al. Selective inhibition of BET bromodomains. Nature 468, 1067-1073 (2010).

37. Huang, C. H. et al. CDK9-mediated transcription elongation is required for MYC addiction in hepatocellular carcinoma. Genes Dev. 28, 1800-1814 (2014).

38. Loven, J. et al. Selective inhibition of tumor oncogenes by disruption of super-enhancers. Cell 153, 320-334 (2013).

39. Lockwood, W. W., Zejnullahu, K., Bradner, J. E. \& Varmus, H. Sensitivity of human lung adenocarcinoma cell lines to targeted inhibition of BET epigenetic signaling proteins. Proc. Natl Acad. Sci. USA 109, 19408-19413 (2012).

40. Hanahan, D. \& Weinberg, R. A. Hallmarks of cancer: the next generation. Cell 144, 646-674 (2011).

41. Lowe, S. W., Cepero, E. \& Evan, G. Intrinsic tumour suppression. Nature 432, 307-315 (2004).

42. Hershko, T. \& Ginsberg, D. Up-regulation of Bcl-2 homology 3 (BH3)-only proteins by E2F1 mediates apoptosis. J. Biol. Chem. 279, 8627-8634 (2004).
43. Chen, S., Dai, Y., Pei, X. Y. \& Grant, S. Bim upregulation by histone deacetylase inhibitors mediates interactions with the Bcl-2 antagonist ABT-737: evidence for distinct roles for Bcl-2, Bcl- $\mathrm{x}_{\mathrm{L}}$, and Mcl-1. Mol. Cell Biol. 29, 6149-6169 (2009).

44. Bean, G. R. et al. PUMA and BIM are required for oncogene inactivationinduced apoptosis. Sci. Signal. 6, ra20 (2013).

45. Corcoran, R. B. et al. Synthetic lethal interaction of combined BCL-XL and MEK inhibition promotes tumor regressions in KRAS mutant cancer models. Cancer Cell 23, 121-128 (2013).

46. Christensen, C. L. et al. Targeting transcriptional addictions in small cell lung cancer with a covalent CDK7 inhibitor. Cancer Cell 26, 909-922 (2014).

47. Barretina, J. et al. The Cancer Cell Line Encyclopedia enables predictive modelling of anticancer drug sensitivity. Nature 483, 603-607 (2012).

48. Morales, A. A. et al. Distribution of Bim determines Mcl-1 dependence or codependence with $\mathrm{Bcl}-\mathrm{x}_{\mathrm{L}} / \mathrm{Bcl}-2$ in Mcl-1-expressing myeloma cells. Blood 118, 1329-1339 (2011).

49. Dai, H. et al. Constitutive BAK activation as a determinant of drug sensitivity in malignant lymphohematopoietic cells. Genes Dev. 29, 2140-2152 (2015).

50. Deng, J. et al. BH3 profiling identifies three distinct classes of apoptotic blocks to predict response to $\mathrm{ABT}-737$ and conventional chemotherapeutic agents. Cancer Cell 12, 171-185 (2007).

\section{Acknowledgements}

We thank AbbVie for providing ABT-263 and ABT-199. We apologize to all the investigators whose research could not be appropriately cited owing to space limitation This work was supported by grants to E.H.C. from the NIH (R01CA125562), Uniting Against Lung Cancer (GC2786) and Cycle for Survival (GC221279). This work was also supported by the NIH P30CA008748.

\section{Author contributions}

A.I.-Y. designed and conducted experiments, and analysed data. E.H.C. designed research, analysed data and supervised the project. P.S.J., H.-C.C., S.H., Y.T.G., S.J. and Y.D. conducted experiments. K.K. analysed data. J.J.H supervised some experiments. K.I., M.C.P., M.D.H. and M.G.K. generated essential reagents.

\section{Additional information}

Supplementary Information accompanies this paper at http://www.nature.com/ naturecommunication

Competing interests: The authors declare no competing financial interests.

Reprints and permission information is available online at http://npg.nature.com/ reprintsandpermissions/

How to cite this article: Inoue-Yamauchi, A. et al. Targeting the differential addiction to anti-apoptotic BCL-2 family for cancer therapy. Nat. Commun. 8, 16078 doi: 10.1038/ ncomms16078 (2017)

Publisher's note: Springer Nature remains neutral with regard to jurisdictional claims in published maps and institutional affiliations.

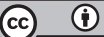

Open Access This article is licensed under a Creative Commons Attribution 4.0 International License, which permits use, sharing, adaptation, distribution and reproduction in any medium or format, as long as you give appropriate credit to the original author(s) and the source, provide a link to the Creative Commons license, and indicate if changes were made. The images or other third party material in this article are included in the article's Creative Commons license, unless indicated otherwise in a credit line to the material. If material is not included in the article's Creative Commons license and your intended use is not permitted by statutory regulation or exceeds the permitted use, you will need to obtain permission directly from the copyright holder. To view a copy of this license, visit http://creativecommons.org/ licenses/by/4.0/

(C) The Author(s) 2017 